August 10, 2018

OITS-682

\title{
Can multi-TeV (top and other) squarks be natural in gauge mediation? $\mathrm{f}$
}

\author{
Kaustubh Agashe ? \\ Institute of Theoretical Science \\ 5203 University of Oregon \\ Eugene OR 97403-5203
}

\begin{abstract}
We investigate whether multi- $\mathrm{TeV}(1-3 \mathrm{TeV})$ squarks can be natural in models of gauge mediated SUSY breaking. The idea is that for some boundary condition of the scalar (Higgs and stop) masses, the Higgs (mass) ${ }^{2}$, evaluated at the renormalization scale $\sim O(100) \mathrm{GeV}$, is not very sensitive to (boundary values of) the scalar masses (this has been called "focussing" in recent literature). Then, the stop masses can be multi-TeV without leading to fine-tuning in electroweak symmetry breaking. Minimal gauge mediation does not lead to this focussing (for all values of $\tan \beta$ and the messenger scale): the (boundary value of) the Higgs mass is too small compared to the stop masses. Also, in minimal gauge mediation, the gaugino masses are of the same order as the scalar masses so that multi-TeV scalars implies multi-TeV gauginos (especially gluino) leading to fine-tuning. We discuss ideas to increase the Higgs mass relative to the stop masses (so that focussing can be achieved) and also to suppress gaugino masses relative to scalar masses (or to modify the gaugino mass relations) in non-minimal models of gauge mediation - then multi-TeV (top and other) squarks can be natural. Specific models of gauge mediation which incorporate these ideas and thus have squarks (and in some cases, the gluino) heavier than a $\mathrm{TeV}$ without resulting in fine-tuning are also studied and their collider signals are contrasted with those of other models which have multi-TeV squarks.
\end{abstract}

\footnotetext{
${ }^{1}$ This work is supported by DOE Grant DE-FG03-96ER40969.

${ }^{2}$ email: agashe@oregon.uoregon.edu
} 


\section{Introduction}

In the supersymmetric extension of the SM, the quantum corrections to the Higgs (mass) ${ }^{2}$ cancel between fermions and bosons in the loops. Thus, SUSY stabilizes the hierarchy between the weak scale and some high energy scale such as the GUT or the Planck scale. SUSY can be broken (softly) so that the superpartners of the SM particles are heavier than the SM particles (as required by phenomenology), but the quadratically divergent corrections to the Higgs $(\text { mass })^{2}$ still cancel. However, with soft SUSY breaking, there are the logarithmically divergent corrections to the Higgs (mass) ${ }^{2}$, proportional to the soft SUSY breaking masses. Due to the large top quark Yukawa coupling, these radiative corrections result in a negative Higgs (mass) ${ }^{2}$ (for the Higgs doublet coupled to the top quark), which, in turn, leads to electroweak symmetry breaking (EWSB) without the need (unlike the SM) to put in a bare negative (mass) ${ }^{2}$ term. "by hand". The expression for $m_{Z}$ (at tree-level) is:

$$
\begin{aligned}
\frac{1}{2} m_{Z}^{2} & =-\mu^{2}+\frac{m_{H_{d}}^{2}-m_{H_{u}}^{2} \tan ^{2} \beta}{\tan ^{2} \beta-1} \\
& \approx-\mu^{2}-m_{H_{u}}^{2}(\text { for large } \tan \beta) .
\end{aligned}
$$

Here, $\mu$ is a supersymmetric mass term for the Higgs, $\tan \beta$ is the ratio of vev's of the two Higgs doublets and $m_{H_{u}}^{2}$ and $m_{H_{d}}^{2}$ are the soft SUSY breaking masses for the Higgs (coupling to the up-type and down-type quarks, respectively) evaluated at the weak scale.

For naturalness of electroweak symmetry breaking i.e., for the $Z$ mass not to be fine-tuned f it is necessary that all the terms on the right-side of Eq. (11) are of order $m_{Z}^{2}$, i.e., the Higgs $(\text { mass })^{2}$ at the weak scale and $\mu^{2}$ are $O(100 \mathrm{GeV})^{2}$. Typically, this implies that top squarks should be lighter than $\sim 1 \mathrm{TeV}$ : heavier top squarks will result in Higgs (mass) ${ }^{2} \sim-\mathrm{TeV}^{2}$ (due to renormalization group ( $R G$ ) scaling to the weak scale and large top quark Yukawa coupling). This will necessitate a large cancellation with $\mu^{2}$ to obtain the correct value of $m_{Z}(\sim 100 \mathrm{GeV})$ (see Eq. (国), i.e., $m_{Z}$ will be very sensitive to $\mu$. Also, in general, if the top squark (stop) mass is heavier than $\sim 1 \mathrm{TeV}$, then $m_{Z}$, in addition to being sensitive to $\mu$, will be very sensitive to the stop mass since it is the stop mass which results in Higgs (mass) ${ }^{2} \sim-\mathrm{TeV}^{2}$ at the weak scale.

Recently, Feng, Matchev, Moroi showed that multi-TeV scalars (in particular top squarks) can be natural in supergravity mediated models with a specific boundary condition (which includes universal scalar masses) for the Higgs and stop masses (at $M_{G U T}$ or $M_{\text {Planck }}$ ) and for $\tan \beta \gtrsim 5$ (with the measured value of the top quark mass) [1]. This happens because, for this boundary condition, the Higgs (mass) ${ }^{2}$ evaluated at the renormalization scale $\sim O(100) \mathrm{GeV}$, is not very sensitive to the boundary value of the scalar masses (these authors call this "focussing"). Then, multi-TeV top squarks will not result in $m_{H_{u}}^{2}$ (at $\left.\sim 100 \mathrm{GeV}\right) \sim-\mathrm{TeV}^{2}$ and thus the stop

\footnotetext{
${ }^{3}$ In general, the $Z$ mass is fine-tuned if it is too sensitive to the fundamental parameters of the theory.
} 
masses can be multi-TeV without leading to fine-tuning in electroweak symmetry breaking. We briefly review this idea in section 2 .

In this paper, we study if a similar focussing of (the weak scale value of) the Higgs (mass) ${ }^{2}$ can occur in models where SUSY breaking is mediated to the MSSM at lower energy scales, i.e., the "messenger" scale is $M_{\text {mess }} \ll M_{\text {Planck }}$ or $M_{G U T}$. The only known mediation of SUSY breaking with $M_{\text {mess }} \ll M_{\text {Planck }}$ or $M_{G U T}$ is by (SM or other) gauge interactions. Gauge mediation (GM) of SUSY breaking (GMSB) does not have the supersymmetric flavor problem (since scalars with the same gauge quantum numbers are degenerate) and also has a predictive spectrum, unlike (generic) supergravity mediation of SUSY breaking. So, we analyse whether in GMSB, the boundary values of the stop and Higgs masses can be such that the Higgs (mass) ${ }^{2}$ at the weak scale is insensitive to the stop masses. Then, multi-TeV stops (and other squarks) can be natural in GMSB f.

In minimal GMSB, a single field which breaks SUSY couples in the superpotential to "messengers" which are vector-like fields in complete multiplets of $S U(5)$. In this minimal model of GM (discussed in section 3), the boundary value of the Higgs mass is too small compared to the stop mass (since scalar masses are proportional to (gauge couplings) ${ }^{2}$, i.e., $\alpha_{A}$ 's) so that focussing does not occur, i.e., the boundary condition necessary for the Higgs (mass) ${ }^{2}$ at the weak scale to be insensitive to the stop mass requires larger Higgs mass compared to the stop mass. Also, in minimal GM, the gaugino masses are comparable to the scalar masses. So, if the scalar masses are multi-TeV, so will be the gaugino (especially gluino) masses which will, in turn, result in large $\left|m_{H_{u}}^{2}\right|$ (through RG scaling) at the weak scale and hence fine-tuning of the $Z$ mass ق.

In section 4 , we study how in (non-minimal) models of GMSB the boundary value of the Higgs mass can be larger so that the boundary condition for the scalar masses which results in focussing can be achieved. We also discuss how to reduce the gaugino masses relative to the scalar masses (or how to modify the relation between the various gaugino masses) so that, even if the scalars are multi-TeV, the gaugino masses do not result in fine-tuning. With focussing and suppression of gaugino masses (or a modified relation between the gaugino masses), multi-TeV $(1-3 \mathrm{TeV})$ squarks can be natural in GMSB.

Some specific non-minimal models of GMSB are analysed in section 5 which have these features so that squarks (and in some models the gluino) can be heavier than a TeV without resulting in fine-tuning in EWSB. We also discuss the collider signals for these models and

\footnotetext{
${ }^{4}$ If scalar and gaugino masses are $s u b-\mathrm{TeV}$, then (for moderate and large $\tan \beta$ ) electric dipole moments of electron and neutron constrain the (relative) phases in gaugino mass and $\mu, B \mu$ terms to be less than $O\left(10^{-2}\right)$ resulting in fine-tuning. With multi-TeV squarks, $O(0.1)$ or larger phases might be allowed, thus (partly) alleviating the problem of fine-tuning of these phases. This could be a motivation for multi-TeV scalars in GM: the scalar degeneracy already solves the supersymmetric flavor problem (even with sub-TeV scalars).

${ }^{5}$ These facts are well known from the studies of minimal GM in the literature.
} 
contrast these to the signals in minimal supergravity models with multi-TeV squarks (and with no fine-tuning due to focussing) [1] and also to the signals in the minimal model of GM with multi-TeV squarks (which is fine-tuned).

\section{Focussing}

We begin with a brief discussion of "focussing", i.e., the boundary condition for the scalar masses such that the Higgs (mass) ${ }^{2}$ at the weak scale is insensitive to the scalar masses. The one-loop renormalization group equations (RGE's) for the (up-type) Higgs and stop masses, neglecting all Yukawa couplings except the top quark Yukawa coupling $\left(\lambda_{t}\right)$ are

$$
\frac{d}{d t}\left(\begin{array}{c}
m_{H_{u}}^{2}(t) \\
m_{\tilde{t}}^{2}(t) \\
m_{\tilde{t}^{c}}^{2}(t)
\end{array}\right)=\left(\begin{array}{c}
3 \\
1 \\
2
\end{array}\right) \frac{\lambda_{t}^{2}(t)}{8 \pi^{2}}\left[m_{H_{u}}^{2}(t)+m_{\tilde{t}}^{2}(t)+m_{\tilde{t}^{c}}^{2}(t)+A_{t}^{2}(t)\right]+\left(\begin{array}{c}
B_{H_{u}}(t) \\
B_{\tilde{t}}(t) \\
B_{\tilde{t}^{c}}(t)
\end{array}\right),
$$

where

$$
B_{i}(t)=-\frac{2}{\pi} \sum_{A} \alpha_{A}(t) C_{A}^{i} M_{A}^{2}(t)
$$

$A_{t}$ is the trilinear soft SUSY breaking term, $M_{A}$ 's are the gaugino masses, $C_{A}^{i}$ are Casimirs for the particle $i$ under the gauge group $A$ and $t \sim \ln \mu_{R G}$ where $\mu_{R G}$ is the renormalization scale. The RGE for the top quark Yukwa coupling is

$$
\frac{d \lambda_{t}(t)}{d t}=\frac{\lambda_{t}(t)}{16 \pi^{2}}\left[6 \lambda_{t}^{2}(t)+\sum_{A=1}^{3} k_{A} g_{A}^{2}(t)\right],
$$

where $\left(k_{3}, k_{2}, k_{1}\right)=(-16 / 3,-3,-13 / 15)$ and $g_{A}$ 's are the gauge couplings. The solution for $m_{H_{u}}^{2}$ can be written as

$$
\begin{aligned}
m_{H_{u}}^{2}(t)= & m_{H_{u}}^{2}(0)\left(\frac{1+I}{2}\right)-\left(\frac{1-I}{2}\right)\left[m_{\tilde{t}}^{2}(0)+m_{\tilde{t}^{c}}^{2}(0)\right] \\
& +a_{M_{3}} M_{3}^{2}(0)+a_{M_{2}} M_{2}^{2}(0)+a_{M_{1}} M_{1}^{2}(0) \\
& +a_{A_{t}} A_{t}^{2}(0)+\sum_{A \neq B} a_{M_{A} M_{B}} M_{A}(0) M_{B}(0)
\end{aligned}
$$

with

$$
\begin{aligned}
I & =\exp \left(\int_{0}^{t} d t^{\prime} \frac{6 \lambda_{t}^{2}\left(t^{\prime}\right)}{8 \pi^{2}}\right) \\
& =1+\frac{6}{8 \pi^{2}} \lambda_{t}^{2}(t) \frac{\int_{0}^{t} d t^{\prime} \prod_{A=1}^{3}\left(\frac{\alpha_{A}\left(t^{\prime}\right)}{\alpha_{A}(0)}\right)}{\prod_{A=1}^{3}\left(\frac{\alpha_{A}(t)}{\alpha_{A}(0)}\right)^{\left(k_{A} / b_{A}\right)}}
\end{aligned}
$$

\footnotetext{
${ }^{6}$ In section 5.2, we comment on the contribution of multi-TeV scalar masses at two-loops to the RGE's.
} 
$t=\ln \left(\mu_{R G} / M_{\text {mess }}\right)$, where $M_{\text {mess }}$ is the messenger scale (the RG scale at which the SUSY breaking is mediated to the MSSM) and $b_{A}$ 's are the gauge beta-functions. Thus, $I$ (and the various $a$ 's) depends on $\lambda_{t}$ (or $\tan \beta$ ), the messenger scale, $M_{\text {mess }}$ and the renormalization scale, $\mu_{R G}$. Typically, $I \sim O(0.1-1)$ and $\left|a_{M_{3}}\right| \sim O(1)>\left|a_{M_{1,2}}\right|$. Assume that the boundary condition is

$$
m_{H_{u}}^{2}(0)=y_{r e q}\left[m_{\tilde{t}}^{2}(0)+m_{\tilde{t}^{c}}^{2}(0)\right]
$$

where

$$
y_{r e q} \equiv \frac{1-I}{1+I}
$$

with $I$ evaluated at the weak scale, i.e., $\mu_{R G} \sim O(100) \mathrm{GeV}$. Then, we see from Eq. (5) that $m_{H_{u}}^{2}$ (at $\sim O(100 \mathrm{GeV})$ ) is independent of the boundary values of the scalar (Higgs and stop) masses since there is a cancellation between the contributions of the (bare) Higgs mass and stop masses - we follow the terminology of [四] and refer to this as "focussing". Thus, as long as this boundary condition is satisfied (in other words, there is focussing), the stop masses can be large (multi-TeV) without resulting in $O(\mathrm{TeV})^{2}\left|m_{H_{u}}^{2}\right|$ at the weak scale. Hence no large cancellation with $\mu^{2}$ is required to obtain the observed $Z$ mass (see Eq. (11)), i.e., the fine-tuning due to $\mu^{2}$ can be small even though the stops are multi-TeV ?. Another fine-tuning in electroweak symmetry breaking is due to the dependence of $m_{H_{u}}^{2}$ (at the weak scale) and hence $m_{Z}$ (see Eq. (1)) on the stop mass. For this fine-tuning to be small, it is necessary, in general, that the stop mass contribution to $m_{H_{u}}^{2}$ at the weak scale be $O(100 \mathrm{GeV})^{2}$. With multi-TeV stop masses, this contribution is (typically) $O(\mathrm{TeV})^{2}$ (see Eq. (可), where $I \sim O(0.1-1)$ ). So, if the stop mass is varied keeping the (bare) Higgs mass fixed, then $m_{Z}$ will be very sensitive to the (multi-TeV) stop mass, even though, due to a cancellation between the contributions (both of which are $\left.O(\mathrm{TeV})^{2}\right)$ of the stop mass and bare Higgs mass, we have $\left|m_{H_{u}}^{2}\right|$ (at the weak scale) $\sim \mu^{2} \sim O\left(m_{Z}^{2}\right)$. However, the sensitivity of $m_{H_{u}}^{2}$ at the weak scale and hence $m_{Z}$ to variations of the (boundary value of) the stop mass (even if it is multi-TeV) will be small as long as the Higgs mass is also varied according to the boundary condition (Eq. (7)) so that the above mentioned cancellation still takes place.

Of course, the tree-level relation of Eq. (1) is modified by radiative corrections. In particular, the one-loop contribution to the effective Higgs potential depends on the stop masses. The oneloop corrected expression for the $Z$ mass is

$$
\begin{aligned}
\frac{1}{2} m_{Z}^{2} & =-\mu^{2}+\frac{m_{H_{d}}^{2}+2 \frac{\partial \Delta V_{1}}{\partial v_{d}^{2}}-\left(m_{H_{u}}^{2}+2 \frac{\partial \Delta V_{1}}{\partial v_{u}^{2}}\right) \tan ^{2} \beta}{\tan ^{2} \beta-1} \\
& \approx-\mu^{2}-m_{H_{u}}^{2}-2 \frac{\partial \Delta V_{1}}{\partial v_{u}^{2}}(\text { for large } \tan \beta),
\end{aligned}
$$

${ }^{7}$ The other (gaugino mass) contributions to $m_{H_{u}}^{2}$ (at the weak scale) (and also, if $\tan \beta$ is small, $m_{H_{d}}^{2}$ at the weak scale) have to be $O(\text { a few } 100 \mathrm{GeV})^{2}$ for $\mu^{2}$ to be $O\left(m_{Z}^{2}\right)$. 
where $\Delta V_{1}$ is the one-loop contribution to the effective potential. Keeping only the stop and top mass contribution in $\Delta V_{1}$ and neglecting the mass mixing of the top squarks, we get

$$
\begin{aligned}
2 \frac{\partial \Delta V_{1}}{\partial v_{u}^{2}}= & \frac{3 \lambda_{t}^{2}}{32 \pi^{2}}\left[-4 m_{t}^{2}\left[\ln \left(\frac{m_{t}^{2}}{\mu_{R G}^{2}}\right)-1\right]\right. \\
& \left.+2\left(m_{\tilde{t}}^{2}+m_{t}^{2}\right)\left[\ln \left(\frac{m_{\tilde{t}}^{2}+m_{t}^{2}}{\mu_{R G}^{2}}\right)-1\right]+2\left(m_{\tilde{t}^{c}}^{2}+m_{t}^{2}\right)\left[\ln \left(\frac{m_{\tilde{t}^{c}}^{2}+m_{t}^{2}}{\mu_{R G}^{2}}\right)-1\right]\right] .
\end{aligned}
$$

Thus, even if the above boundary condition for the Higgs and stop masses (Eq. (7)) is satisfied, $m_{Z}$ will still depend (weakly) on the stop masses.

It turns out that for the measured value of the top quark (pole) mass, $m_{t}=173.8 \pm 5.2 \mathrm{GeV}$ [2] and for $\tan \beta \gtrsim 5, I \approx 1 / 3$ for RG scaling from the GUT or Planck scale to the weak scale [1]. This means that for the following boundary condition in supergravity mediated SUSY breaking (where the messenger scale is the GUT or Planck scale), "focussing" results:

$$
m_{H_{u}}^{2}(0) \approx \frac{1}{2}\left[m_{\tilde{t}}^{2}(0)+m_{\tilde{t}^{c}}^{2}(0)\right] .
$$

This class of models includes the minimal supergravity model where there is a universal scalar mass $\left(m_{0}\right)$ [1]. Hence, in minimal supergravity and for these values of $\tan \beta$ and $m_{t}$, scalars can be multi-TeV without leading to fine-tuning of $m_{Z}$.

It is clear from the expression for $I$ that for smaller $\tan \beta$ and hence larger $\lambda_{t}, I$ will be smaller and hence $y_{\text {req }}>1 / 2$ (for $M_{\text {mess }} \approx M_{G U T}$ ). Thus, even for small $\tan \beta$, there is a boundary condition for the scalar masses which leads to focussing in supergravity mediated SUSY breaking so that, even for small $\tan \beta$, stop masses can be multi-TeV without resulting in fine-tuning. The required value of $y$ is shown in Fig. 1 as a function of $\tan \beta$ where we see that $y_{\text {req }} \approx 1 / 2$ for $\tan \beta \gtrsim 5$.

\section{Minimal Gauge Mediation}

We now consider lower messenger scales, $M_{\text {mess }} \ll M_{\text {Planck }}$, to see if "focussing" can take place. In Figs.2 and 3, the required $y$ (Eq. (8)) is plotted as a function of the messenger scale for $\tan \beta=10$ and as a function of $\tan \beta$ for a fixed messenger scale.

Gauge mediation is the only known mechanism to mediate SUSY breaking to the MSSM at these energy scales. So, we study the scalar mass predictions in gauge mediation to see if the boundary condition required for focussing can be satisfied. Typically in models of gauge mediation, a SUSY breaking field $X$ (with non-zero vev's in its scalar and $F$-components) couples

\footnotetext{
${ }^{8}$ Of course, there is a lower limit on $\sin \beta(\tan \beta)$ of $\sim 0.85$ (1.6) if we impose the condition that $\lambda_{t}$ should not reach it's Landau pole below the GUT scale.
} 


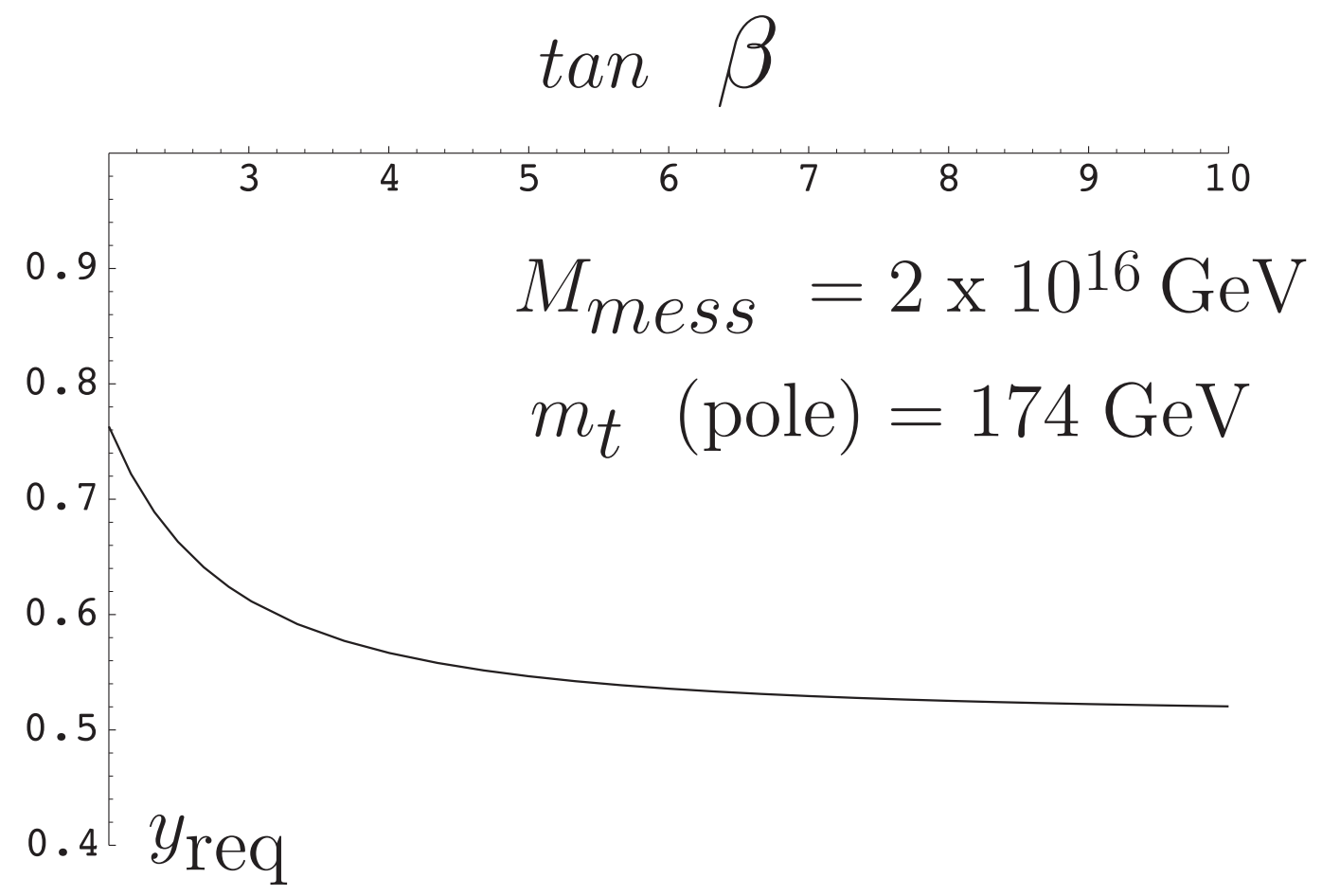

Figure 1: The ratio of the Higgs mass to the stop mass required for focussing (see Eq. (8)) as a function of $\tan \beta$ with the messenger scale $\sim 2 \times 10^{16} \mathrm{GeV}$. The renormalization scale is $\mu_{R G} \approx 1$ $\mathrm{TeV}$ and the top quark (pole) mass is $174 \mathrm{GeV}$. 


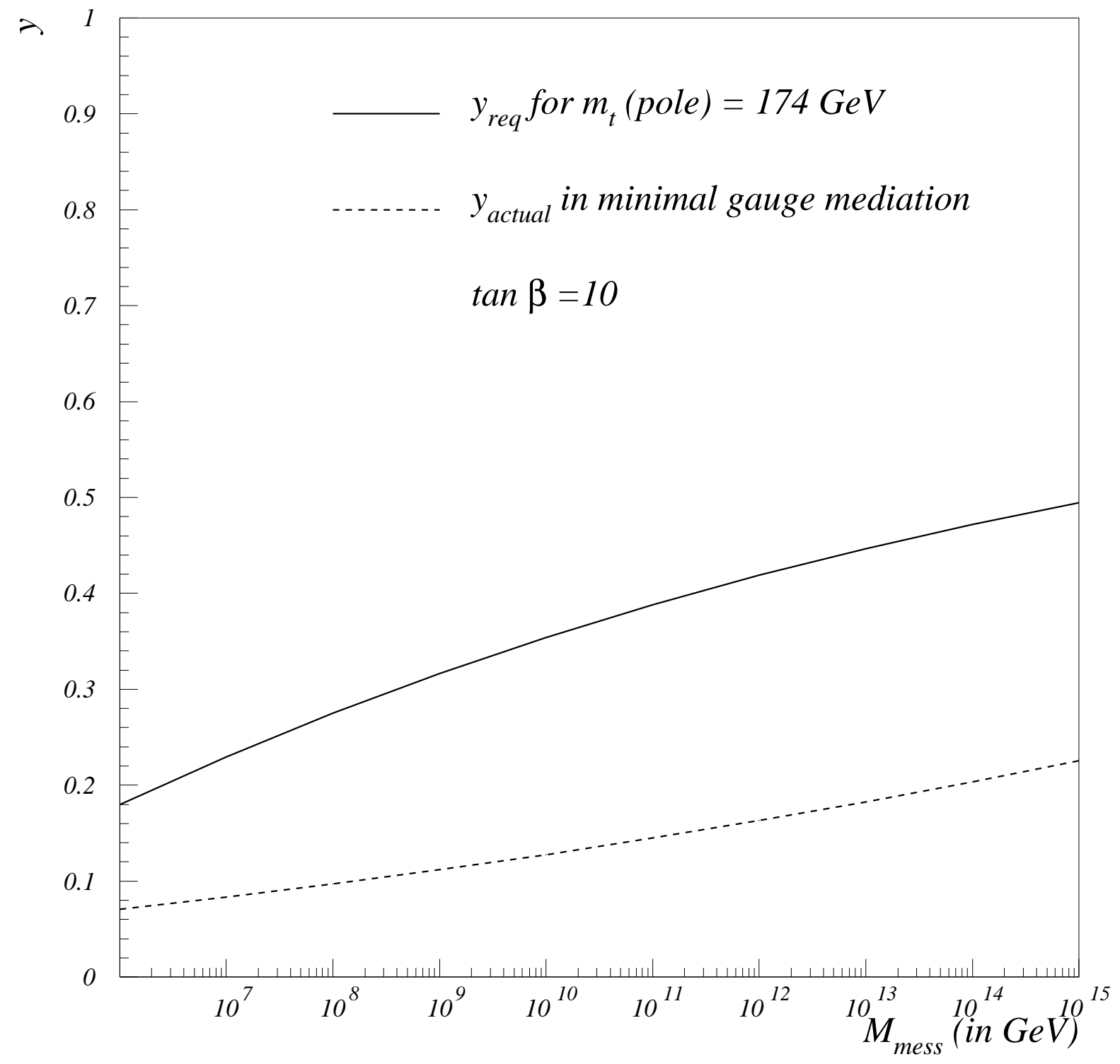

Figure 2: The ratio of the Higgs mass to the stop mass required for focussing (solid line) (see Eq. (8)) as a function of the messenger scale, $M_{\text {mess }}$. The renormalization scale is $\mu_{R G} \approx 1 \mathrm{TeV}$, $\tan \beta=10$ and the top quark (pole) mass is $174 \mathrm{GeV}$. The dashed line is the actual ratio of the Higgs mass to the stop mass in the minimal model of gauge mediation (see Eq. (15)). 


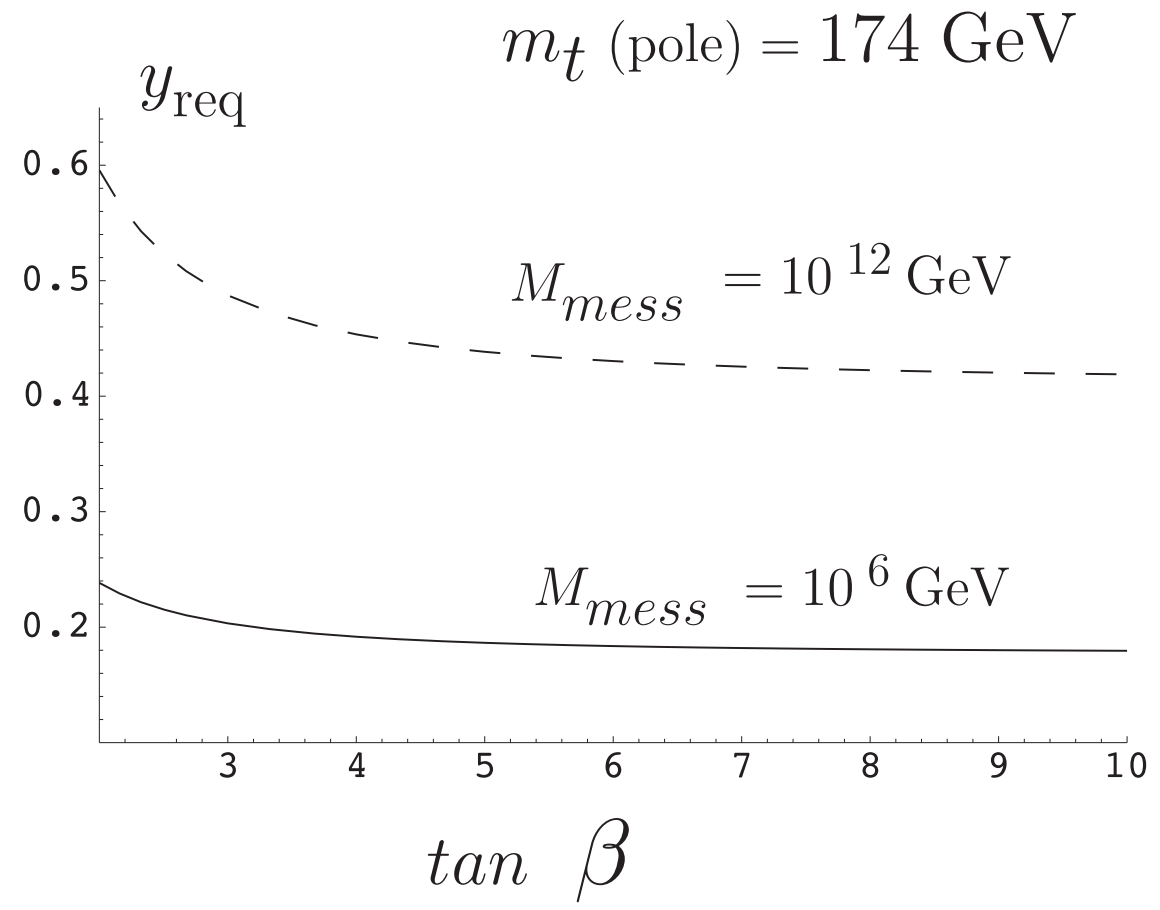

Figure 3: The ratio of the Higgs mass to the stop mass required for focussing (see Eq. (8)) as a function of $\tan \beta$ for messenger scale $M_{\text {mess }}=10^{6} \mathrm{GeV}$ (solid line) and $M_{\text {mess }}=10^{12} \mathrm{GeV}$ (dashed line). The renormalization scale is $\mu_{R G} \approx 1 \mathrm{TeV}$ and the top quark (pole) mass is 174 $\mathrm{GeV}$. 
to "messenger" fields which are vector-like under the SM gauge group. When these messenger fields are integrated out at the scale $M_{\text {mess }} \sim X$, soft SUSY breaking masses are generated for the MSSM scalars $\left(m_{i}^{2}\right)$ and gauginos $\left(M_{A}\right)$ :

$$
\begin{gathered}
m_{\text {scalar } i^{2}}^{2}=2\left(\frac{F_{X}}{X}\right)^{2}\left[\sum_{m e s s, A} C_{A}^{i} N_{m e s s}^{A}\left(\frac{\alpha_{A}(X)}{4 \pi}\right)^{2}\right]\left[1+O\left(\frac{F_{X}}{X^{2}}\right)\right], \\
M_{A}=\frac{\alpha_{A}(X)}{4 \pi} \frac{F_{X}}{X} \sum_{m e s s} N_{m e s s}^{A}\left[1+O\left(\frac{F_{X}}{X^{2}}\right)\right]
\end{gathered}
$$

where $N_{\text {mess }}^{A} \delta^{a b}=\operatorname{tr}\left(T^{a} T^{b}\right)$, T's are the generators of the gauge group in the representation of the messengers and $C_{A}^{i}$ is the Casimir of the scalar particle $i$ under the gauge group $A$.

To get an idea of focussing in a model, we define

$$
y_{\text {actual }} \equiv \frac{m_{H_{u}}^{2}(0)}{m_{\tilde{t}}^{2}(0)+m_{\tilde{t}^{c}}(0)} .
$$

In a minimal model of gauge mediation, a single SUSY breaking field couples to messenger fields which are in complete multiplets of $S U(5)$ so that we get

$$
y_{\text {actual }}=\frac{\frac{3}{4} \alpha_{2}^{2}(0)+\frac{3}{20} \alpha_{1}^{2}(0)}{\frac{8}{3} \alpha_{3}^{2}(0)+\frac{3}{4} \alpha_{2}^{2}(0)+\frac{17}{60} \alpha_{1}^{2}(0)} .
$$

In Fig.2, we also plot $y_{\text {actual }}$ (which depends only on $M_{\text {mess }}$ ) for the minimal GM model: we see that for all $M_{\text {mess }}, y_{\text {actual }}<y_{r e q}$. For smaller $\tan \beta, \lambda_{t}$ is larger and hence $y_{r e q}$ is larger. So, we see that for all $\tan \beta$ and $M_{\text {mess }}$, minimal GM does not give the boundary condition required for "focussing" of $m_{H_{u}}^{2}$.

As mentioned in the introduction and as can be seen from Eqs. (12) and (13), in typical models of GM (in particular, the minimal model), the scalar and gaugino masses are comparable so that multi-TeV scalars would imply multi-TeV gauginos. In the minimal model, $M_{3}(0)>$ $M_{2}(0), M_{1}(0)$ (for $\left.M_{\text {mess }} \ll M_{G U T}\right)$. Thus, multi-TeV gauginos (especially gluino) will result in $m_{H_{u}}^{2} \sim-\mathrm{TeV}^{2}$ (see Eq. (5)), where typically $a_{M_{3}}<0$ and for $M_{\text {mess }}$ not too small, $\left|a_{M_{3}}\right| \sim$ $\left.O(1)>\left|a_{M_{1,2}}\right|\right)$ and thus fine-tuning of $m_{Z}$.

Typically in GM (certainly in the minimal model), $m_{H_{u}}^{2} \approx m_{H_{d}}^{2}$ at $M_{m e s s}$ so that large ((multi$\left.\mathrm{TeV})^{2}\right) m_{H_{u}}^{2}$ at $M_{\text {mess }}$ implies that $m_{H_{d}}^{2}$ is also large. For $\tan \beta \gtrsim 5, m_{Z}$ is very insensitive to $m_{H_{d}}^{2}$ (at the weak scale) (see Eq. (四) so that even if it is $\sim(\mathrm{TeV})^{2}$, there is no fine-tuning, i.e., no large cancellation with $\mu^{2}$ is required (as long as $m_{H_{u}}^{2}$ at the weak scale is not too large). However, if $\tan \beta$ is small and the Higgs masses are $\sim \mathrm{TeV}$ at $M_{\text {mess }}$, then at the weak scale,

\footnotetext{
${ }^{9}$ As mentioned in the footnote on page 2, there has been extensive analysis of EWSB in minimal GM from which it is clear that multi-TeV squarks will lead to fine-tuning. We have redone (reworded) this analysis so that the modifications to minimal GM required to achieve "focussing" are transparent (see below).
} 
$m_{H_{d}}^{2} \sim(\mathrm{TeV})^{2}$ also (since RG scaling results in a positive contribution to $m_{H_{d}}^{2}$ due to gaugino masses - the Yukawa couplings of $H_{d}$ are small for small $\tan \beta$ ); this results in fine-tuning in EWSB (see Eq. (11), where $m_{H_{d}}^{2} \sim \mathrm{TeV}^{2}$ and $\tan \beta$ is small).

Thus, in order to have multi-TeV squarks without resulting in fine-tuning in models of GM, we require:

1. additional contribution to the Higgs (mass) ${ }^{2}$ (or a non-minimal messenger sector) so that yactual (see Eq. (14)) can be larger and thus focussing can be achieved. Also, either $\tan \beta \gtrsim 5$ so that a large $\left(\sim(\mathrm{TeV})^{2}\right) m_{H_{d}}^{2}$ (at the weak scale) does not lead to fine-tuning or if $\tan \beta$ is small, then the additional contribution to the Higgs (mass) ${ }^{2}$ should be such that $m_{H_{d}}^{2}$ at the boundary is small $\left(O(100 \mathrm{GeV})^{2}\right)$ even though $m_{H_{u}}^{2}$ is larger

and

2. suppression of the gaugino masses relative to the scalar masses so that even if the scalars are multi- $\mathrm{TeV}$, the gauginos can be sub- $\mathrm{TeV}$ and hence not result in fine-tuning or

a modification of the relation between the gaugino masses so that the gaugino mass contribution to the Higgs (mass) $)^{2}$ can be smaller (say due to cancellation between the gluino and wino masses) even if the gauginos are heavy.

\section{Non-Minimal Gauge Mediation}

\section{$4.1 \quad$ Increasing $m_{H_{u}}^{2}(0)$}

We discuss two ways to increase the Higgs mass relative to the stop mass (at the messenger scale) in GMSB so that $y_{\text {actual }} \approx y_{\text {req }}$.

1. The Higgs potential in the MSSM has the parameters $B \mu$ and $\mu$ in addition to the Higgs soft (mass) $)^{2}$. The parameter $B \mu$ breaks SUSY and both $\mu$ and $B \mu$ break the $U(1)_{P Q}$ symmetry. In the minimal model of GM, $B \mu$ is zero at the messenger scale and a non-zero $B \mu$ (typically small) is induced in RG scaling. In generic models of GM, there are additional interactions of the Higgs fields with the SUSY breaking sector to (break the $U(1)_{P Q}$ symmery and) generate the $B \mu$ term (at $M_{\text {mess }}$ ) and also a $\mu$ term. Typically the models which generate the $\mu$ term have $\mu \approx 0$ in the supersymmetric limit so that the $\mu$ term is generated once SUSY is broken. Then, it's value is related to the soft masses and is therefore close to the weak scale as required by naturalness.

These additional interactions can also generate extra contributions to the Higgs soft (mass) ${ }^{2}$ $\left(m_{H_{u}}^{2}\right.$ and $\left.m_{H_{d}}^{2}\right)$ [3]. In other words, there is effectively an extra parameter, $m_{H_{u}}^{2}(0)$ in these models which is independent of the stop masses (which are unchanged) 四. If we choose this

\footnotetext{
${ }^{10}$ The "effective" $m_{H_{u}}^{2}(0)=m_{H_{u}}^{2}(0)$ due to GM (this part is related to the stop masses) $+m_{H_{u}}^{2}(0)$ due to
} 
parameter so that $y_{\text {actual }} \approx y_{\text {req }}$ 円 then the Higgs (mass) ${ }^{2}$ at the weak scale will be (almost) independent of the stop masses, i.e., it will be $O(100 \mathrm{GeV})^{2}$ even if the stop masses are multi$\mathrm{TeV}$. In other words, for each $\tan \beta$ (or equivalently $\lambda_{t}$ ) and $M_{\text {mess }}$ (i.e., for each $y_{\text {req }}$ ) there is a value of $m_{H_{u}}^{2}(0)$ which results in focussing [1]

Of course, this requires a "coincidence", i.e., a correlation between the additional Higgs interactions which generate $m_{H_{u}}^{2}(0)$ (the GM contribution to $m_{H_{u}}^{2}(0)$ relative to the stop masses is fixed for a given messenger sector) and the messenger scale and/or $\lambda_{t}$ (since $y_{r e q}$ depends on the messenger scale and $\lambda_{t}$ ). This means that, with (boundary) scalar masses $\sim$ multi-TeV, if these additional Higgs couplings (and hence only $\left.m_{H_{u}}^{2}(0)\right)$ are changed by $O(1)$, then the Higgs $(\text { mass })^{2}$ at the weak scale changes by $O(\mathrm{TeV})^{2}$ so that $m_{Z}$ is very sensitive to changes in these couplings. On the other hand, if $m_{S U S Y} \sim \alpha /(4 \pi) F_{X} / X$ which determines the size of the soft masses for the MSSM sparticles is changed by $O(1)$ (with the additional Higgs couplings fixed), then both the Higgs and the stop masses change such that $y_{\text {actual }}$ remains the same and thus $m_{Z}$ is insensitive to changes in $m_{S U S Y}$ even if $m_{S U S Y}$ is multi-TeV (of course, provided $y_{\text {actual }} \approx y_{\text {req }}$ ).

This situation is similar to the case of focussing in supergravity mediated SUSY breaking with universal scalar masses (for $\tan \beta \gtrsim 5$ ) [四]. As long as the Higgs and the stop masses are varied keeping the boundary relation between the masses the same as the one which results in focussing (for example, by changing the scale of SUSY breaking), $m_{Z}$ is not very sensitive to these masses, even if they are multi-TeV. But, there is, a priori (in the absence of flavor or other symmetries), no reason for scalar masses to be universal (or to satisfy the particular boundary condition) in supergravity so that in generic supergravity models it should be possible to vary the stop mass keeping the (bare) Higgs mass fixed. As discussed earlier, in this case, if the stop mass is multi-TeV, then $m_{Z}$ is very sensitive to it (even though the Higgs (mass) ${ }^{2}$ at the weak scale may be $\left.O(100 \mathrm{GeV})^{2}\right)$.

2. If the number of messenger fields charged under $S U(2)_{\text {weak }} / U(1)_{Y}$ (messenger "leptons") is larger than those charged under $S U(3)_{\text {color }}$ (messenger "quarks"), then the Higgs mass (relative to the stop masses) will be larger (see Eq. (12)). Other possibilities to increase $y_{\text {actual }}$ are to have a smaller supersymmetric mass ( $X$ in Eq. (12) ) for the messenger leptons (see section additional Higgs interactions.

${ }^{11}$ As mentioned earlier, even if $y_{a c t u a l}=y_{r e q}, m_{Z}$ will still be (weakly) sensitive to the stop mass due to the one-loop contribution to the effective Higgs potential, Eq. (10). We can define an "effective" $y_{r e q}$ to include this effect.

${ }^{12}$ The additional Higgs interactions generate $\mu, B \mu$ and a contribution to $m_{H_{u, d}}^{2}$ (in addition to the GM contribution). Typically (see, for example, the model of Dvali, Giudice, Pomarol in [3]) $\mu, B \mu$ and (a part of) $m_{H_{u, d}}^{2}$ are given in terms of the SUSY breaking scale (say, $m_{S U S Y}$ ) and more than two additional Higgs couplings so that the two minimization conditions for the Higgs potential (Eqs. (9) and (18)) are not sufficient to fix the values of these couplings (for given $\tan \beta, M_{\text {mess }}$ and $m_{S U S Y}$ ) and hence $m_{H_{u}}^{2}(0)$ (i.e., $y_{\text {actual }}$ ) is a free parameter.

${ }^{13}$ As mentioned earlier, if $\tan \beta$ is small, then the additional Higgs soft masses should be such that $m_{H_{d}}^{2} \sim$ $O(\text { a few } 100 \mathrm{GeV})^{2}$ at $M_{\text {mess }}$. 
5.1 for a specific model) or to have a larger SUSY breaking mass $\left(F_{X}\right.$ in Eq. (12) $)$ for the messenger leptons (see section 5.3 for a specific model). In the case where the numbers (or the supersymmetric masses) of the messenger quarks and leptons are not equal, unification of the SM gauge couplings will be affected. Unlike the case 1 above, the particular boundary relation between the Higgs and the stop masses is guaranteed by the content of the messenger sector, i.e., once the messenger sector is fixed, we (typically) cannot vary the Higgs and stop masses independently.

\subsection{Reducing the fine-tuning due to gaugino masses}

In this section, we discuss some methods to reduce the gaugino mass contribution to the Higgs $(\text { mass })^{2}$ at the weak scale in models of GM so that even if the scalars are multi-TeV, the gaugino masses will not lead to fine-tuning in EWSB.

1. It is possible that the gaugino masses are generated by a set of messenger quarks and leptons which are different from those that generate the scalar masses. This means that there can be an extra (independent of the scalar masses) parameter for gaugino masses. For example, in the model of Dobrescu [4], there is one set of messengers which are SM singlets, but charged under $U(1)_{(B-L)}$ gauge group. SUSY breaking is mediated to the MSSM squarks and sleptons, but not to the MSSM gauginos (and the Higgs fields), by $(B-L)$ gauge interactions. There is another set of messengers which are vector-like under the SM gauge group. This second set of messengers gives contributions to both scalar and gaugino masses which depend on different (superpotential) couplings (and also might be smaller by a loop factor) than the $U(1)_{(B-L)}$ contribution (to scalar masses) 四. Thus, effectively, there are two parameters, one for the gaugino masses and one for the scalar masses so that even if scalars are multi-TeV, the gauginos can be light.

2. The gaugino masses break $U(1)_{R}$ symmetry so that generically the gaugino mass generated at one-loop by integrating out messenger quarks and leptons is given by $\sim \alpha /(4 \pi) F M_{R} / M_{\text {mess }}^{2}$, where $F, M_{\not R}$ and $M_{\text {mess }}$ are, respectively, the SUSY breaking, $R$-symmetry breaking and messenger scales. In minimal models of gauge mediation, $M_{\not R} \sim M_{\text {mess }}$ so that there is no suppression of the gaugino masses relative to the scalar masses (which do not break the $R$-symmetry). But, in non-minimal models, it is possible that $M_{\not R} \ll M_{\text {mess }}$ so that (even if the same messengers generate scalar and gaugino masses) the gaugino masses are suppressed by the ratio of $R$-symmetry breaking scale to the messenger scale. Thus, again, there is effectively an extra (independent) paramater for gaugino masses. An example of this idea is shown in section 5.3 as part of a specific model.

\footnotetext{
${ }^{14}$ In [5] also, there is $U(1)_{(B-L)}$ gauge mediation (which generates SUSY breaking masses for squarks and sleptons only) in addition to mediation by SM gauge group (which is necessary to generate MSSM gaugino masses), but both these contributions are related and hence gaugino masses are not independent of scalar masses.
} 
3. As in case 2 of section 4.1, if the number of messenger leptons is more than the number of quarks or their masses (supersymmetric or SUSY breaking) are different, then the wino mass relative to gluino mass can increase (see Eq. (13)). In this case, the net gaugino mass contribution to the Higgs (mass) $)^{2}$ is not too large in magnitude (i.e., it can be $O(\text { a few } 100 \mathrm{GeV})^{2}$ ) even if the gauginos are heavy (say $\sim \mathrm{TeV}$ ) (see Eq. (5)), where $a_{M_{3}}<0, a_{M_{2}}>0$ and $\left|a_{M_{3}}\right|>\left|a_{M_{2}}\right|$ ). In other words, if the ratio of wino mass to gluino mass is larger, then there can be a (partial) cancellation between wino and gluino mass contributions to $m_{H_{u}}^{2}$ at the weak scale. A concrete model with 3 pairs of messenger leptons and 1 pair of quarks is analysed in section 5.2 .

4. For very low messenger scales, $\left|a_{M_{A}}\right|$ 's in Eq. (5) are small so that gauginos can be heavy without resulting in large $\left|m_{H_{u}}^{2}\right|$ at the weak scale and hence fine-tuning.

For electroweak symmetry breaking to occur, (typically) we require $m_{H_{u}}^{2}$ (at the weak scale) to be negative: either the scalar mass or gaugino mass contribution to $m_{H_{u}}^{2}$ at the weak scale has to be negative. Thus, too much "focussing" (i.e., too small scalar mass contribution) and too much suppression of the gaugino masses (especially gluino relative to wino) will not result in EWSB. For multi-TeV squarks to be natural, it is not necessary that the scalar mass contribution to $m_{H_{u}}^{2}$ at the weak scale vanish exactly: EWSB is natural as long as both the scalar and gaugino mass contributions are $O(\text { a few } 100 \mathrm{GeV})^{2}$ and at least one of them is negative so that $m_{H_{u}}^{2} \sim-O(100 \mathrm{GeV})^{2}$. It is also possible that both the contributions are $O(\mathrm{TeV})^{2}$ (again, with at least one of them negative), but there is a cancellation so that $m_{H_{u}}^{2} \sim-O(\text { a few } 100 \mathrm{GeV})^{2}$.

\section{$5 \quad$ Specific Models}

We now study concrete models of gauge mediation which incorporate the ideas of section 1 and thus have multi-TeV squarks without leading to fine-tuning in EWSB.

\subsection{Raby model}

In this model [10, 11], the messenger quarks have a (supersymmetric) mass $\sim M_{G U T}$ 囫 whereas (some of) the messenger leptons have a smaller (supersymmetric) mass, $M \sim 10^{15} \mathrm{GeV}$. The $R$-symmetry breaking scale, $M_{R}$, is $\sim M$. Thus, the gluino mass is suppressed relative to the wino mass (and left-handed slepton mass) by $\left(M / M_{G U T}\right)^{2}$ : one factor of $M / M_{G U T}$ is due to the larger messenger scale $\left(M_{\text {mess }} \sim M_{G U T}\right)$ for quarks and the other is due to $M_{\not R}$ being smaller than $M_{\text {mess }}$ (whereas for wino mass, $M_{\text {mess }} \sim M_{\not R} \sim M$ ). As mentioned earlier, this means that even if the left-handed slepton/squark masses are multi-TeV, the gluino can be lighter. Also, the right-handed stop (and other squark) (mass) ${ }^{2}$ is suppressed by $\left(M / M_{G U T}\right)^{2}$ relative to the

\footnotetext{
${ }^{15}$ This model implements the Dimopoulos-Wilczek doublet-triplet splitting mechanism [12]: a pair of messenger quarks has a Dirac mass with the pair of Higgs triplets due to the vev of the $S O(10)$ adjoint field while the Higgs doublets are massless.
} 
left-handed squark/slepton (mass) ${ }^{2}$ due to the larger supersymmetric mass for messenger quarks. This increases $y_{\text {actual }}$ as compared to minimal models of GM. Thus, this model has the features required to have multi- $\mathrm{TeV}$ squarks without resulting in fine-tuning.

However, as shown in [11], it turns out that the suppression of the gluino mass and the righthanded stop mass is too much if $M \sim 10^{15} \mathrm{GeV}$ or smaller: one problem is that both the gaugino and the scalar mass contributions to the (up-type) Higgs (mass) ${ }^{2}$ at the weak scale tend to be positive (wino mass is large relative to gluino mass and $y_{\text {actual }}$ is too large) so that it is hard to get EWSB (in particular, $\mu^{2}$ tends to be too small or negative unless $\tan \beta$ is small while for small $\tan \beta$, the lightest Higgs mass is too small). The other problem is that right-handed stop (mass) ${ }^{2}$ is driven negative by the top quark Yukawa coupling in RG scaling to the weak scale since it's boundary value is suppressed compared to the Higgs and left-handed stop mass and also the light gluino does not give a large enough positive contribution. If the supergravity contribution to the soft masses (which is $O\left(F_{X} / M_{\text {Planck }}\right)$, but with, in general, arbitrary coefficients) is to be smaller than the GM contribution, we need $M \lesssim 1 / 10 M_{G U T} \sim 10^{15} \mathrm{GeV}$; otherwise, the soft mass spectrum is not predictive. Thus, it is hard to make this model phenomenologically viable in the desired region of parameter space.

In [11], these problems were alleviated by adding a $U(1) D$-term contribution to the soft scalar masses - the additional contribution is negative (positive) for the Higgs (squark) soft (mass) $)^{2}$. However, this reduces $y_{\text {actual }}$ and thus "focussing" is hard to achieve.

\subsection{More messenger leptons than quarks}

As mentioned earlier (see Eqs. (12) and (13)), if the number of messenger leptons is larger than messenger quarks, then (a) yactual is larger so that the magnitude of the scalar mass contribution to $m_{H_{u}}^{2}$ at the weak scale decreases and (b) the wino mass (relative to the gluino mass) increases, thus decreasing the (magnitude) of the gaugino mass contibution to $m_{H_{u}}^{2}$ at the weak scale as well. Then, the squarks and some of the gauginos can be heavier than $1 \mathrm{TeV}$ without leading to fine-tuning in EWSB. A model of GM with 3 pairs of messenger leptons, denoted by $l+\bar{l}$, and 1 pair of messenger quarks, denoted by $q+\bar{q}$, where $q+l$ form a $\mathbf{5}$ of $S U(5)$, and with a low messenger scale $\sim 100 \mathrm{TeV}$ was used in [6] to reduce the fine-tuning in EWSB. Here, we consider the same model, but with higher messenger scales also. The soft mass spectrum at the messenger scale is (assuming $\sqrt{F_{X}} \ll X$ ):

$$
\begin{gathered}
m_{\text {Scalar } i \approx 2\left(\frac{F_{X}}{X}\right)^{2}[}\left[C_{3}^{i}\left(\frac{\alpha_{3}}{4 \pi}\right)^{2}+3 C_{2}^{i}\left(\frac{\alpha_{2}}{4 \pi}\right)^{2}+\frac{3}{5} Y_{i}^{2} \frac{11}{5}\left(\frac{\alpha_{1}}{4 \pi}\right)^{2}\right], \\
M_{3} \approx \frac{\alpha_{3}}{4 \pi} \frac{F_{X}}{X} \\
M_{2} \approx 3 \frac{\alpha_{2}}{4 \pi} \frac{F_{X}}{X}
\end{gathered}
$$




$$
M_{1} \approx \frac{11}{5} \frac{\alpha_{1}}{4 \pi} \frac{F_{X}}{X}
$$

where the gauge couplings are evaluated at $M_{\text {mess }} \sim X$.

With the above messenger sector (i.e., 3 pairs of leptons and 1 pair of quarks) and the MSSM particle content, the three gauge couplings do not unify at $\sim 10^{16} \mathrm{GeV}$. However, in [6], with a messenger scale $\sim 100 \mathrm{TeV}$, color triplets were added with a mass of $\sim \mathrm{TeV}$ so that coupling unification can be maintained $\square$. Here, we do not introduce any additional quarks to preserve coupling unification.

In this model, all the gaugino and the scalar masses can be written in terms of only one parameter - we choose this parameter to be the gluino mass at the messenger scale, $M_{3}(0)$. Thus, $y_{\text {actual }}$ is not useful to determine if we can have focussing, i.e., both the scalar mass and gaugino mass contibutions to $m_{H_{u}}^{2}$ at the weak scale depend on $M_{3}(0)$. We assume that there is a mechanism to generate $\mu$ and $B \mu$ terms and that this mechanism does not give additional contributions to the Higgs soft masses. We also assume that no trilinear $A$-term is generated at the messenger scale (as in the minimal model of GM). Thus, the fundamental parameters of this model are $M_{3}(0), M_{\text {mess }}, \lambda_{t}, \mu$ and $B \mu$ : these parameters are constrained by the measured values of $m_{Z}$ and $m_{t}$ so that there are 3 free (or input) parameters which we choose to be $M_{3}(0)$, $M_{\text {mess }}$ and $\tan \beta$. The values of $\mu$ and $B \mu$ are determined (in terms of $\tan \beta$ and $M_{3}(0)$ ) from the minimization conditions, Eqs. (9) and (18) (see below), for the Higgs potential.

$$
\sin 2 \beta=\frac{2 B \mu}{2 \mu^{2}+m_{H_{u}}^{2}+m_{H_{d}}^{2}} .
$$

For the analysis in this and the next section, we use one-loop RGE's to evolve the scalar and gaugino masses and the dimensionless couplings from $M_{\text {mess }}$ to the weak scale. The one-loop contribution to the effective Higgs potential (Eq. (10)) is taken into account. If the scalar soft masses are $1-3 \mathrm{TeV}$ at $M_{\text {mess }}$, then, in RG scaling to the weak scale, the two-loop contribution (due to the heavy scalar masses) to the Higgs and the squark/stop (mass) ${ }^{2}$ (depending on $M_{\text {mess }}$ ) can be $O(100 \mathrm{GeV})^{2}$ (it is smaller for the Higgs (mass) ${ }^{2}$ than the stop (mass $\left.)^{2}\right)^{\boxplus}$. This contribution is typically positive for the Higgs (mass) ${ }^{2}$ (due to the effect of $\lambda_{t}$ ) and thus improves the fine-tuning, i.e., reduces $c$ defined below by $O(1)$ and so does not change the orderof-magnitude estimate of fine-tuning. The two-loop contribution to the the squark/stop (mass) ${ }^{2}$ is negative, but since the squark/stop (mass) ${ }^{2}$ after one-loop RG evolution to the weak scale is $\sim O(\mathrm{TeV})^{2}$ (for multi-TeV boundary scalar masses), the two-loop effect is not significant.

We use the sensitivity of $m_{Z}$ to a fundamental parameter, $\lambda_{i}$, as a measure of fine-tuning due

\footnotetext{
16 These exotic "quarks" were used to generate $\mu$ and $B \mu$ terms with the addition of a gauge singlet. Also, this particle content could be embedded in a GUT model with a doublet-triplet splitting mechanism [6].

${ }^{17}$ For numerical estimates of this two-loop contribution, see, for example, 7 .
} 
to that parameter [8]:

$$
c\left(m_{Z}^{2} ; \lambda_{i}\right) \equiv \frac{\lambda_{i}}{m_{Z}^{2}} \frac{\partial m_{Z}^{2}}{\partial \lambda_{i}} .
$$

It has been argued that a large value of $c\left(m_{Z}^{2} ; \lambda_{i}\right)$ does not necessarily imply that $m_{Z}$ is fine-tuned [9]. However, in this paper, we are interested in order-of-magnitude estimates of fine-tuning only and so we use

$$
c \equiv \operatorname{Max}\left[\left|c\left(m_{Z}^{2} ; \mu^{2}\right)\right|,\left|c\left(m_{Z}^{2} ; m_{S U S Y}^{2}\right)\right|\right],
$$

where $m_{S U S Y}$ is a SUSY breaking parameter in the MSSM, as a measure of fine-tuning: $c \gtrsim$ 100 signals unnaturalness. If the stops are multi- $\mathrm{TeV}$, then the fine-tuning due to the top quark Yukawa coupling $\left(c\left(m_{Z}^{2} ; \lambda_{t}\right)\right)$ (with $\lambda_{t}$ evaluated at $M_{\text {mess }}$ ) is large since the stop mass contribution to $m_{H_{u}}^{2}$ at the weak scale (and hence to $m_{Z}^{2}$ ) is $O\left(\mathrm{TeV}^{2}\right.$ ) (even if $m_{H_{u}}^{2}$ (at the weak

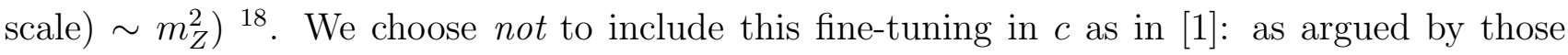
authors, it is possible that the top quark Yukawa coupling reaches a "fixed point" in RG scaling from say the Planck scale to the GUT scale or $M_{\text {mess }}$ so that the sensitivity to $\lambda_{t}\left(M_{P l}\right)$ (which is the fundamental parameter) may not be large. Also $\lambda_{t}$ is not related to SUSY breaking - we are interested mainly in the fine-tuning due to the SUSY breaking parameters (we include $\mu$ in this since in many models, $\mu$ is related to SUSY breaking).

If Figs. 1 and 5, we show contours of fine-tuning in this model. The renormalization scale, $\mu_{R G}$, is chosen to be $2 M_{3}(0) \approx \sqrt{m_{\tilde{t}_{1}} m_{\tilde{t}_{2}}}$ (where $m_{\tilde{t}_{1,2}}$ are the physical stop masses) $\$$.

The right-handed squark (excluding the stop) masses (at the weak scale) are $\approx 2 M_{3}(0)$ whereas the left-handed squarks (including the stop) are heavier, where $M_{3}(0)$ is the gluino mass at the messenger scale. The right-handed stop (to be precise, the lighter stop eigenstate) mass is (depending on $\left.M_{\text {mess }}\right)(1-2) \times M_{3}(0)$, i.e., it is smaller than the other squarks (due to RG scaling to the weak scale with a large Higgs mass at the boundary). The right-handed slepton mass is (depending on $\left.M_{\text {mess }}\right)(1 / 2-3 / 2) \times M_{3}(0)$. Gaugino masses at the weak scale can be obtained (in terms of $M_{3}(0)$ ) from Eq. (17) using $M_{A}(t) \propto \alpha_{A}(t)$ (at one-loop).

From these figures, we see that even for $M_{3}(0) \gtrsim 500 \mathrm{GeV}$, it is possible to have $c \lesssim 100$ for a wide range of messenger scales, especially for values of $m_{t}$ (slightly) larger than the central value (but within $\sim 1 \sigma$ of the central value). Also, for some values of the other parameters, the finetuning for $1.5 \mathrm{TeV} \gtrsim M_{3}(0) \gtrsim 500 \mathrm{GeV}$ can be almost as good as for $200 \mathrm{GeV} \lesssim M_{3}(0) \lesssim 500$

\footnotetext{
18 To be precise, $m_{H_{u}}^{2}$ (at the weak scale) $\sim-O(\text { a few } 100 \mathrm{GeV})^{2}$ due to a cancellation between $\sim O\left(\mathrm{TeV}^{2}\right)$ contributions of the Higgs and stop masses. Thus, if $\lambda_{t}$ is varied by (say) a few percent, then this cancellation is no longer as effective so that $m_{H_{u}}^{2}$ (and hence $m_{Z}^{2}$ ) changes by $O(1)$. This can be seen from Figs. (4 and 8 where we see that $c \sim \mu^{2} / m_{Z}^{2} \sim m_{H_{u}}^{2} / m_{Z}^{2}$ is very sensitive to $m_{t}$ (for a fixed $\tan \beta$ ) or in other words $m_{H_{u}}^{2}$ at the weak scale is very sensitive to $\lambda_{t}$.

${ }^{19}$ With the one-loop contribution to the Higgs potential included, the results will not be very sensitive to the RG scale.
} 
$\mathrm{GeV}$ (the lower limit on $M_{3}(0)$ is from collider searches for right-handed sleptons and stops) ${ }^{20}$. Thus, multi-TeV ( $\sim 1-3 \mathrm{TeV}$ ) squarks (including the left-handed stop) can be natural in this model: discovery of these sparticles at the LHC might be difficult (at least with $10(\mathrm{fb})^{-1}$ of integrated luminosity). For $M_{3}(0) \lesssim 1 \mathrm{TeV}$ the right-handed stop mass can be $\lesssim 1 \mathrm{TeV}$ so that it is likely to be detected at the LHC. For $M_{3}(0) \gtrsim 500 \mathrm{GeV}$, (depending on $M_{\text {mess }}$ ) the gluino mass (at the weak scale) can be $\gtrsim 1 \mathrm{TeV}$ so that it might not be detected at the LHC with $10(\mathrm{fb})^{-1}$ of integrated luminosity. The wino mass (at the weak scale) is about the same as the gluino mass, but for the region of parameter space with $c \lesssim 100$, the $\mu$ term is $\lesssim 600$ $\mathrm{GeV}$ so that even if the wino mass is $\sim 1 \mathrm{TeV}$ or more, the lighter chargino/neutralino will still be detected at a (1 or 1.5) TeV lepton collider (or at the LHC, if $\mu$ is very small), even though the heavier chargino/neutralino will escape detection. The right-handed slepton masses, even for $M_{3}(0) \gtrsim 500 \mathrm{GeV}$ (which leads to squarks heavier than $\sim 1 \mathrm{TeV}$ ), can be lighter than $\sim 500-750 \mathrm{GeV}$ so that their discovery is possible at a $\sqrt{s}=1$ or $1.5 \mathrm{TeV}$ lepton collider 巴. The lightest SM superpartner is typically the neutralino which has bino as the dominant component since $M_{1} \sim 0.4 M_{3}$ (at the weak scale).

The results presented for $\tan \beta=10$ are also valid (roughly) for $5 \lesssim \tan \beta \lesssim 20$ since in this range of $\tan \beta, \lambda_{t}$ does not change much while the bottom and tau Yukawa couplings are small. For smaller values of $\tan \beta$ and $M_{3}(0) \gtrsim 500 \mathrm{GeV}$, the fine-tuning will be worse than for large $\tan \beta$ since then $m_{H_{d}}^{2} \sim(\mathrm{TeV})^{2}$ (in this model, the two Higgs masses are the same at $M_{m e s s}$ ).

For very low messenger scales (and with multi-TeV squarks) so that the RG logarithm is small and for $N_{l} \gg N_{q}$, it is hard to get $m_{H_{u}}^{2}$ (at the weak scale) $<0$ since the positive wino mass and (bare) Higgs mass contributions to $m_{H_{u}}^{2}$ at the weak scale dominate. This can be seen in Fig.5 where the region with very small $M_{\text {mess }}\left(\sim 10^{6} \mathrm{GeV}\right)$ and $M_{3}(0) \gtrsim 500 \mathrm{GeV}$ is excluded since it has $\mu^{2}<0$.

\subsection{Dimopoulos, Thomas, Wells model}

In this model (appendix of [13), the messengers in complete $S U(5)$ multiplets 22 couple to a SUSY breaking field which is an adjoint of $S U(5)$ rather than a singlet whereas the supersymmetric mass of the messengers comes from an $S U(5)$ singlet field. This modifies the mass spectrum for the gauginos and scalars.

The superpotential is:

$$
W=\lambda_{3} X q_{1} \bar{q}_{1}+\lambda_{2} X l_{1} \bar{l}_{1}+X \xi^{2}
$$

\footnotetext{
${ }^{20}$ For example, for values of $m_{t}$ about $1 \sigma$ larger than the central value, we see in Fig. 1 that the fine-tuning contours are sort of parallel to the $M_{3}(0)$ axis.

${ }^{21}$ As mentioned earlier, right-handed sleptons are lighter than squarks (by a factor $\sim \alpha_{1} / \alpha_{3}$ with $\alpha$ 's evaluated at $M_{\text {mess }}$ ) as in typical models of GM.

${ }^{22}$ Unlike the previous model, this model preserves gauge coupling unification.
} 


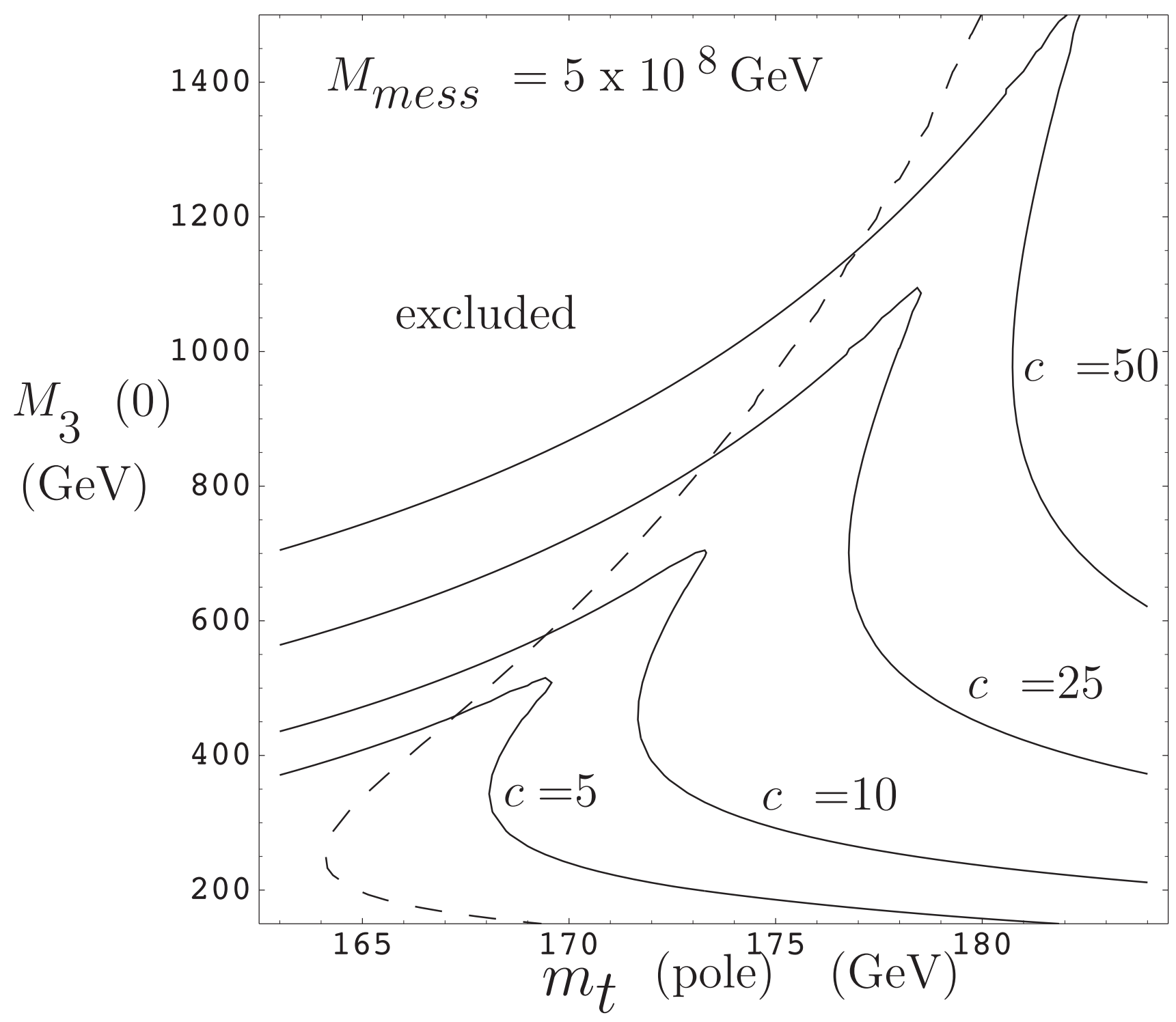

Figure 4: The fine-tuning $c$ (Eq. (20)) (solid lines) in the model of GM with 3 pairs of messenger leptons and 1 pair of messenger quarks (section 5.2) as a function of the gluino mass at the messenger scale, $M_{3}(0)$, and the top quark (pole) mass, for $\tan \beta=10$. The messenger scale is $\approx 5 \times 10^{8} \mathrm{GeV}$. The region to the left of the dashed line has chargino mass smaller than $90 \mathrm{GeV}$ or $\mu^{2}<0$ and is therefore excluded. 


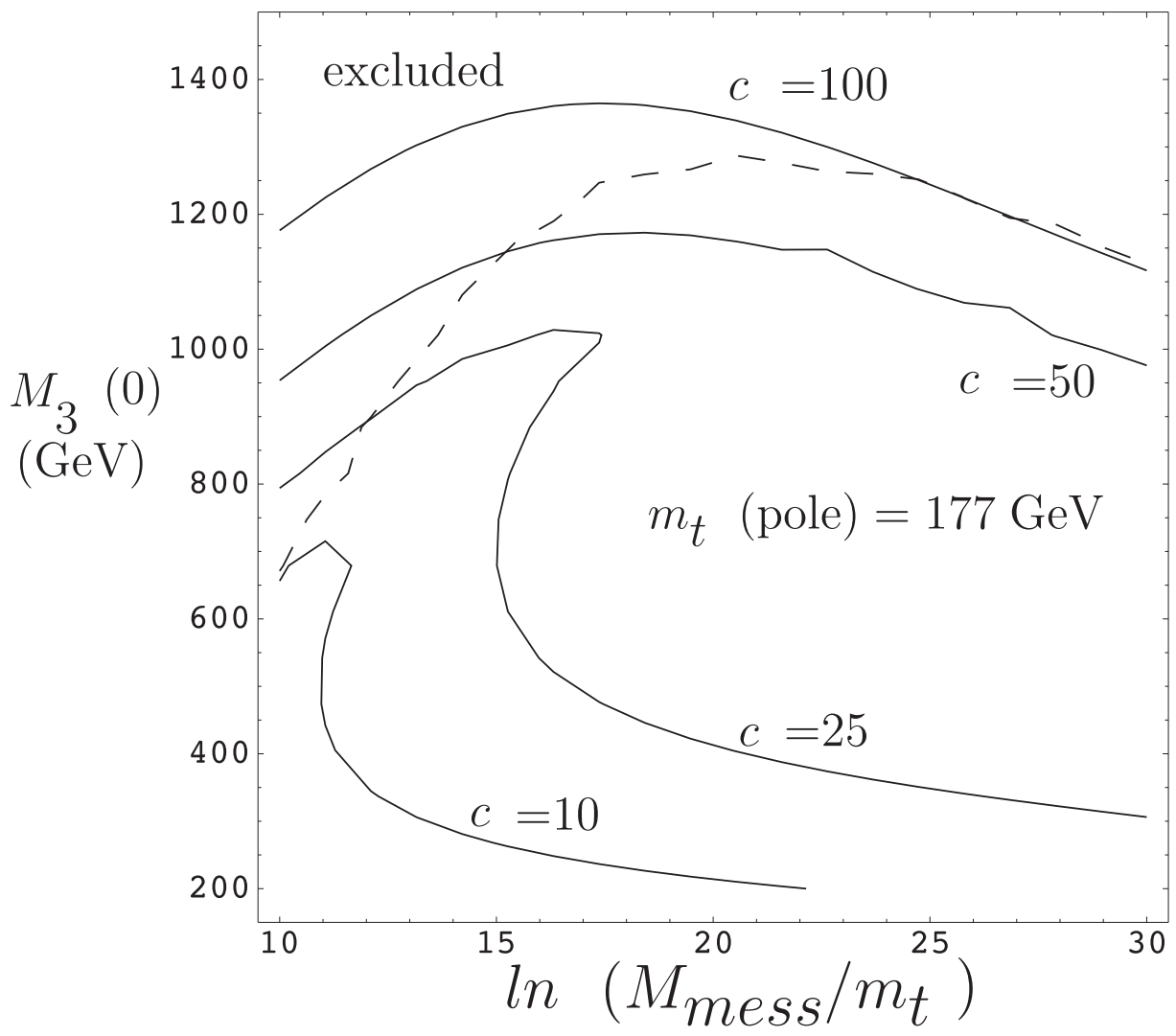

Figure 5: The fine-tuning $c$ (Eq. (20)) (solid lines) in the model of GM with 3 pairs of messenger leptons and 1 pair of messenger quarks (section 5.2) as a function of the gluino mass at the messenger scale, $M_{3}(0)$, and the messenger scale, $M_{\text {mess }}$, for $m_{t}$ (pole) $=177 \mathrm{GeV}$ and $\tan \beta=10$. $\ln \left(M_{\text {mess }} / m_{t}\right)=10$ corresponds to $M_{\text {mess }} \approx 4 \times 10^{6} \mathrm{GeV}$ and $\ln \left(M_{\text {mess }} / m_{t}\right)=30$ corresponds to $M_{\text {mess }} \approx 2 \times 10^{15} \mathrm{GeV}$. The region above the dashed line has chargino mass smaller than 90 $\mathrm{GeV}$ or $\mu^{2}<0$ and is therefore excluded. 


$$
+\lambda_{3}^{\prime} S\left(q_{1} \bar{q}_{2}+q_{2} \bar{q}_{1}\right)+\lambda_{2}^{\prime} S\left(l_{1} \bar{l}_{2}+l_{2} \bar{l}_{1}\right)
$$

where $X$ is an a adjoint of $S U(5), S$ is a gauge singlet and $q, l$ form a $\mathbf{5}$ of $S U(5)$. With $F_{X} \neq 0$, $F_{S} \sim 0$ and $\lambda^{\prime} S \gg \lambda X$ ? , the $R$-symmetry breaking scale is $\sim X$ and we get the following mass spectrum when the messengers are integrated out (assuming $\sqrt{F_{X}} \ll S$ )

$$
\begin{aligned}
m_{\text {Scalar } i}^{2} \approx & 2\left(\frac{F_{X}}{S}\right)^{2}\left[C_{3}^{i}\left(\frac{\lambda_{3}}{\lambda_{3}^{\prime}}\right)^{2}\left(\frac{\alpha_{3}}{4 \pi}\right)^{2}+C_{2}^{i}\left(\frac{\lambda_{2}}{\lambda_{2}^{\prime}}\right)^{2}\left(\frac{\alpha_{2}}{4 \pi}\right)^{2}\right. \\
& \left.+\frac{3}{5} Y_{i}^{2}\left(\frac{\alpha_{1}}{4 \pi}\right)^{2}\left(\frac{2}{5}\left(\frac{\lambda_{3}}{\lambda_{3}^{\prime}}\right)^{2}+\frac{3}{5}\left(\frac{\lambda_{2}}{\lambda_{2}^{\prime}}\right)^{2}\right)\right] \\
M_{1} & \sim \frac{\alpha_{1}}{4 \pi} \frac{F_{X} X}{S^{2}}\left[\frac{2}{5} \frac{\lambda_{3}^{2}}{\lambda_{3}^{\prime 2}}+\frac{3}{5} \frac{\lambda_{2}^{2}}{\lambda_{2}^{\prime 2}}\right] \\
M_{2} & \sim \frac{\alpha_{2}}{4 \pi} \frac{F_{X} X}{S^{2}} \frac{\lambda_{2}^{2}}{\lambda_{2}^{\prime 2}} \\
M_{3} & \sim \frac{\alpha_{3}}{4 \pi} \frac{F_{X} X}{S^{2}} \frac{\lambda_{3}^{2}}{\lambda_{3}^{\prime 2}}
\end{aligned}
$$

where the gauge and the Yukawa couplings are evaluated at the messenger scale $\sim \lambda^{\prime} S$. Since the field $X$ is an $S U(5)$ adjoint whereas $S$ is a singlet, we get

$$
\begin{aligned}
& \frac{\lambda_{2}}{\lambda_{3}}=-\frac{3}{2} \\
& \lambda_{2}^{\prime}=\lambda_{3}^{\prime}
\end{aligned}
$$

at the GUT scale. If we assume that $\lambda, \lambda^{\prime} \ll g_{A}$ (where $g_{A}$ 's are the gauge couplings), then $\lambda_{3} / \lambda_{3}^{\prime}$ and $\lambda_{2} / \lambda_{2}^{\prime}$ are RG invariant (below the GUT scale). This implies that $\left(\lambda_{2} / \lambda_{2}^{\prime}\right)\left(\lambda_{3}^{\prime} / \lambda_{3}\right) \approx-3 / 2$ at the messenger scale also so that the mass spectrum at the messenger scale is:

$$
\begin{aligned}
m_{\text {Scalar } i^{2} \approx 2 \Lambda^{2}}^{2}\left[C_{3}^{i}\left(\frac{\alpha_{3}}{4 \pi}\right)^{2}+C_{2}^{i} \frac{9}{4}\left(\frac{\alpha_{2}}{4 \pi}\right)^{2}+\frac{21}{20} Y_{i}^{2}\left(\frac{\alpha_{1}}{4 \pi}\right)^{2}\right], \\
M_{1} \sim \frac{\alpha_{1}}{4 \pi} \Lambda x \frac{7}{4}, \\
M_{2} \sim \frac{\alpha_{2}}{4 \pi} \Lambda x \frac{9}{4}, \\
M_{3} \sim \frac{\alpha_{3}}{4 \pi} \Lambda x,
\end{aligned}
$$

\footnotetext{
${ }^{23}$ With this superpotential, the vev's of $X$ and $S$ are undetermined at tree-level, but with a modified superpotential and/or radiative corrections, the hierarchy of the vev's can be achieved.

${ }^{24}$ Under the $R$-symmetry which is not broken by the vev of $S$, but is broken by the vev of $X$, the $R$-charges are $\left(X, S, l_{1}, \bar{l}_{1}, l_{2}, \bar{l}_{2}\right)=(2,0, x,-x, 2+x, 2-x)$.

${ }^{25}$ This model also has "gauge" messengers: the gauge multiplet corresponding to the broken generators of $S U(5)$ has a non-supersymmetric spectrum (due to $F_{X} \neq 0$ and $X$ being an adjoint) and couples to the MSSM sparticles. However, the contribution of the "gauge" messengers to the soft masses is $\sim \alpha /(4 \pi) F_{X} / M_{G U T}$ which is much smaller than the contribution of the $q, l$ messengers which is $\sim \alpha /(4 \pi) F_{X} / S$ (assuming $S \lesssim 1 / 10 M_{G U T}$ ).
} 
where $\Lambda \equiv \lambda_{3} F_{X} /\left(\lambda_{3}^{\prime} S\right)$ and $x \equiv\left(\lambda_{3} X\right) /\left(\lambda_{3}^{\prime} S\right)$. We see that the gaugino masses can be suppressed relative to the scalar masses due to $R$-symmetry breaking scale being smaller than the messenger scale, i.e., by a factor $\sim \lambda X /\left(\lambda^{\prime} S\right)$. Thus, there is an independent parameter for the gaugino masses so that gauginos can be sub-TeV even if the scalars are multi-TeV. Also, the SUSY breaking mass for messenger leptons is larger (due to $\lambda_{2}>\lambda_{3}$ ) than that for the quarks so that yactual is larger.

Thus, the soft SUSY breaking masses can be written in terms of two parameters which we choose to be the gluino mass at the messenger scale, $M_{3}(0)$, and the right-handed up-squark mass at the messenger scale, $m_{\tilde{u}^{c}}(0)$ to

In Fig.6, we plot $y_{\text {actual }}$ (which depends only on $M_{\text {mess }}$ for a given model: see Eq. (14)) for this model as a function of the messenger scale and also $y_{r e q}$ for $\tan \beta=10$ and (since we want stops to be multi-TeV) the renormalization scale $\mu_{R G} \approx 2 \mathrm{TeV}$. We see that $y_{\text {actual }} \approx y_{\text {req }}$, especially for smaller values of $m_{t}$ and very low or high messenger scales, so that we expect focussing in this model (certainly, focussing is "better" than in minimal GM: compare Figs.2 and 6) .

In Figs.7, 8 and 9, we plot the contours of fine-tuning for this model. The renormalization scale, $\mu_{R G}$, is chosen to be $m_{\tilde{u}^{c}}(0) \approx \sqrt{m_{\tilde{t}_{1}} m_{\tilde{t}_{2}}}$ (where $m_{\tilde{t}_{1,2}}$ are the physical stop masses). As mentioned above and as can be seen in Fig.8, focussing $\left(y_{\text {actual }} \approx y_{\text {req }}\right)$ is "better" for smaller values of $m_{t}$. So, in Figs.7 and 9 we use $m_{t}$ (pole) $=166.3 \mathrm{GeV}$, which is $\sim 1 \sigma$ below the central value.

The right-handed squark (other than stop) masses at the weak scale are $\approx m_{\tilde{u}^{c}}(0)$ whereas the left-handed squarks (including the stop) are (slightly) heavier. The right-handed stop mass (at the weak scale) is typically (depending on $\left.M_{\text {mess }}\right)(1 / 2-1) \times m_{\tilde{u}^{c}}(0)$ (due to the effect of the top quark Yukawa coupling and the larger value of $m_{H_{u}}^{2}(0)$ than in minimal GM). The right-handed slepton mass is $\sim(1 / 4-3 / 4) \times m_{\tilde{u}^{c}}(0)$ (again, depending on $M_{\text {mess }}$ ). Thus, there is a lower limit of $\sim 500 \mathrm{GeV}$ on $m_{\tilde{u}^{c}}(0)$ from collider searches for right-handed sleptons and stops. Gaugino masses can be obtained (in terms of $M_{3}(0)$ ) from Eq. (26) using $M_{A}(t) \propto \alpha_{A}(t)$ (at one-loop).

We see from the figures that even for $m_{\tilde{u}^{c}}(0) \sim 1-3 \mathrm{TeV}$, it is possible to have $c \lesssim 100$ for a wide range of $M_{\text {mess }}$ and for smaller values of $m_{t}(\sim 1 \sigma$ below the central value). Also, for these values of $m_{t}$ and either very low $\left(\sim 10^{7} \mathrm{GeV}\right)$ or high $\left(\sim 10^{15} \mathrm{GeV}\right)$ messenger scales, some values of $m_{\tilde{u}^{c}}(0)$ in the range $\sim 1-3 \mathrm{TeV}$ are almost as natural as sub-TeV $m_{\tilde{u}^{c}}(0)$ च.

${ }^{26}$ The fundamental parameters are $M_{3}(0), m_{\tilde{u}^{c}}(0), \lambda_{t}, \mu, B \mu$ and $M_{\text {mess }}$ and the free (or input) parameters are chosen to be $M_{3}(0), m_{\tilde{u}^{c}}(0), \tan \beta$ and $M_{\text {mess }}$. As before, $\mu$ and $B \mu$ are determined from the minimization conditions.

${ }^{27}$ For example, we see in Fig. 8 that for $m_{t}$ about $1 \sigma$ below the central value, the fine-tuning contours are sort of parallel to the $m_{\tilde{u}^{c}}$ axis. However, for larger values of $m_{t}$ and for intermediate $M_{\text {mess }}\left(\sim 10^{12} \mathrm{GeV}\right)$, since focussing is not so good (see Fig.6), sub-TeV $m_{\tilde{u}^{c}}(0)$ is more natural than $m_{\tilde{u}^{c}}(0) \gtrsim 1 \mathrm{TeV}$, even though it is still 


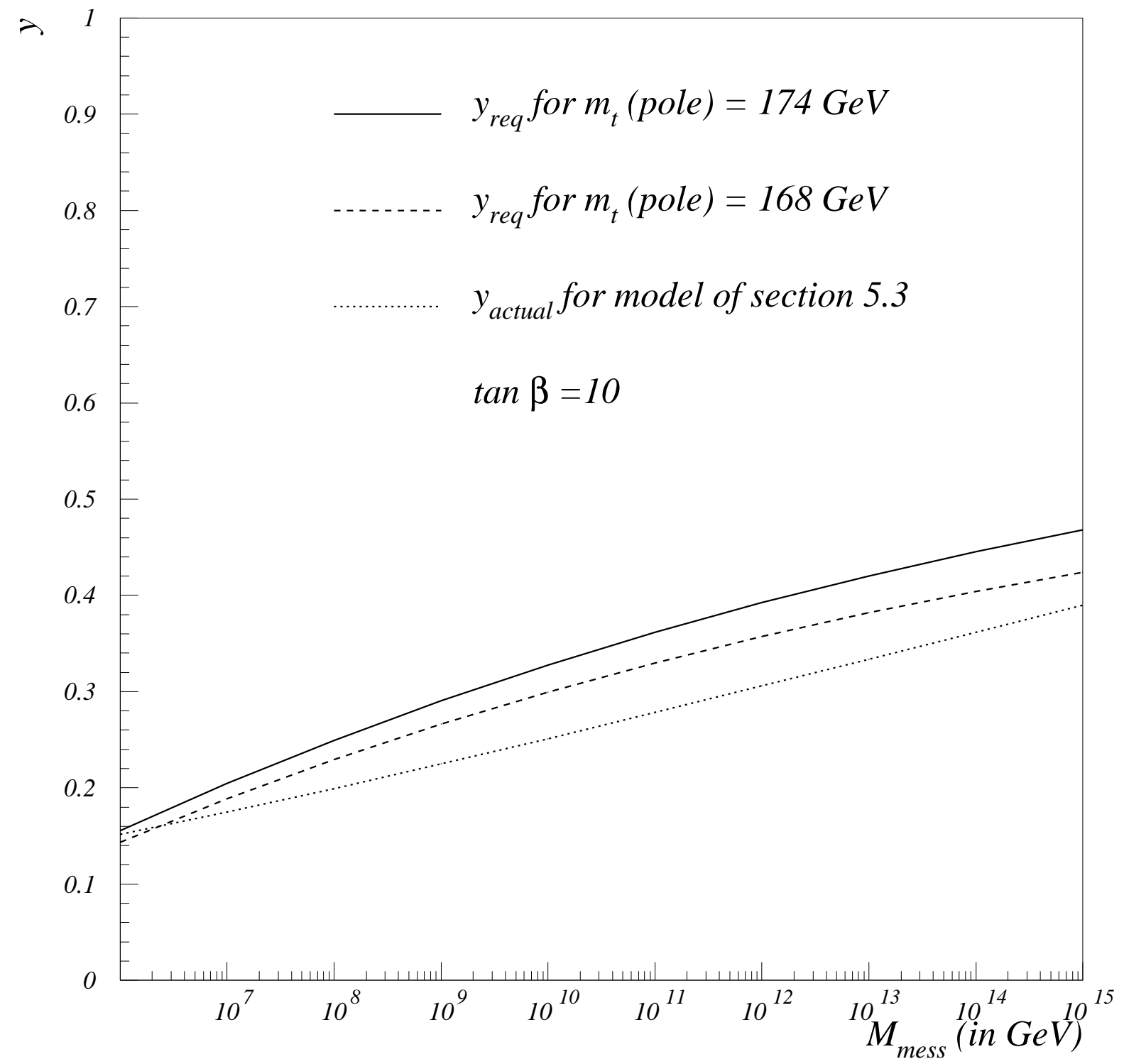

Figure 6: The ratio of the Higgs mass to the stop mass (Eq. (14)) in the Dimopoulos, Thomas, Wells model (section 5.3) (dotted line) as a function of messenger scale. The solid and the dashed lines are the ratios of Higgs mass to the stop mass required for focussing (Eq. (8)) for $m_{t}$ (pole) $=174 \mathrm{GeV}$ and $168 \mathrm{GeV}$, respectively, with $\tan \beta=10$ and the renormalization scale $\mu_{R G} \approx 2$ $\mathrm{TeV}$. 
Thus, multi-TeV $(\sim 1-3 \mathrm{TeV})$ squarks (including left-handed stop) can be natural in this model. If $m_{\tilde{u}^{c}}(0) \lesssim 1.5 \mathrm{TeV}$, then the right-handed slepton can be discovered at a $\mathrm{TeV}$ lepton collider. The right-handed stop can be lighter than $1 \mathrm{TeV}$ if $m_{\tilde{u}^{c}}(0) \lesssim 2 \mathrm{TeV}$ and so is likely to be detected at the LHC whereas the other squarks might escape detection at the LHC (at least with $10(\mathrm{fb})^{-1}$ of integrated luminosity) if $m_{\tilde{u}^{c}}(0) \gtrsim 1 \mathrm{TeV}$.

Since there is an independent parameter for the gaugino masses, it is possible that all the gauginos will be detected at the $\mathrm{LHC} / \mathrm{TeV}$ lepton collider provided $M_{3}(0) \lesssim 500 \mathrm{GeV}$. From Eq. (26), we see that the wino mass is enhanced as compared to minimal GM (i.e., $M_{2} / M_{3} \sim 1$ at the weak scale) thereby reducing the gaugino mass contribution to the Higgs (mass) ${ }^{2}$ (as discussed earlier) so that $c \lesssim 100$ is possible even for $M_{3}(0) \gtrsim 500 \mathrm{GeV}$ (see Fig.()). In fact, for some values of the other parameters, $M_{3}(0) \gtrsim 500 \mathrm{GeV}$ is as natural as $M_{3}(0) \lesssim 500 \mathrm{GeV}$. In this case, depending on $M_{\text {mess }}$, we can have wino and gluino masses at the weak scale $\sim 1 \mathrm{TeV}$ or heavier (without any fine-tuning) and thus the wino will be beyond the reach of a $\mathrm{TeV}$ linear collider and the detection of gluino at the LHC might require more than $10(\mathrm{fb})^{-1}$ of integrated luminosity. As before, for the region of the parameter space with $c \lesssim 100$, we have $\mu \lesssim 600$ $\mathrm{GeV}$ so that the Higgsinos (to be precise the neutralino and chargino which have Higgsinos as the dominant component) can be discovered at a (1 or 1.5) TeV lepton collider also (or at the LHC, if $\mu$ is very small). Since $M_{1} \sim 1 / 3 M_{3}$ (at the weak scale) in this model, the neutralino which has bino as the dominant component is (typically) the lightest SM superpartner.

As mentioned earlier, for smaller values of $\tan \beta$ (say less than 5 and for a fixed $m_{t}$ ) $y_{\text {req }}$ is larger so that "focussing" will not be effective in this model 2 . However, the above results are also valid for $5 \lesssim \tan \beta \lesssim 20$ (since the top quark Yukawa coupling is roughly the same and the bottom quark Yukawa coupling is small in this range of $\tan \beta$ ).

The spectrum in the above two GMSB models (sections 5.2 and 5.3) (with multi-TeV squarks) 29 should be contrasted to the spectrum in supergravity mediation with multi-TeV universal scalar mass (and focussing so that multi-TeV stops are natural) 四. In this supergravity model, in addition to squarks, (right-handed) slepton masses are also multi- $\mathrm{TeV}$ and hence will escape detection at $\mathrm{TeV}$ lepton colliders (whereas, as mentioned earlier, in GMSB models right-handed sleptons are lighter than squarks by a factor $\sim \alpha_{1} / \alpha_{3}$ ) and gaugino masses are $s u b-\mathrm{TeV}$ so that there is no fine-tuning (whereas in the models of sections 5.2 and 5.3 , gluino mass can be $\sim 1$ $\mathrm{TeV}$ or heavier without resulting in fine-tuning). Also, for $M_{\text {mess }} \lesssim 10^{7} \mathrm{GeV}$ in the models of sections 5.2 and 5.3, the intrinsic scale of SUSY breaking, $\sqrt{F}$, can be less than $\sim 10^{3} \mathrm{TeV}$ $\left(F_{X} / M_{\text {mess }} \sim 100 \mathrm{TeV}\right.$ to give $\sim \mathrm{TeV}$ squarks). In this case, it is possible (unlike in supergravity possible to have $c \lesssim 100$ for $m_{\tilde{u}^{c}}(0) \gtrsim 1 \mathrm{TeV}$ and these values of $m_{t}$ and $M_{\text {mess }}$.

${ }^{28}$ Also, in this model, multi-TeV squarks implies $m_{H_{d}}^{2} \sim(\mathrm{TeV})^{2}$ leading to fine-tuning for small $\tan \beta$.

${ }^{29} \mathrm{We}$ have not been very precise about the mass spectrum and detectability of sparticles at colliders in these two models. A detailed analysis (including two-loop RGE's) of fine-tuning and mass spectrum (phenomenology) in these models is in progress. 
mediation) that the lightest SM superpartner, i.e., the next-to-lightest supersymmetric particle (NLSP) (the gravitino is LSP if $M_{\text {mess }} \ll M_{G U T}$ ) will decay inside the detector (after being produced directly or in cascade decays). The NLSP decays give the well-studied $\not p_{T}+\gamma \gamma$ (or $Z Z$ ) (if the NLSP is a neutralino) and $\not p_{T}+2$ leptons (if the NLSP is a right-handed slepton) signals of GM.

Minimal GM with the SUSY breaking scale $m_{S U S Y} \sim \alpha /(4 \pi) \Lambda \gtrsim 1 \mathrm{TeV}$ can also have multi-TeV squarks (and gluino) and possibly a wino mass $\sim 1 \mathrm{TeV}$ and a sub-TeV (right-handed) slepton so that its signals might mimic those in the models of sections 5.2 and 5.3 . However, due to focussing, the models of sections 5.2 and 5.3 have $\mu \lesssim 500 \mathrm{GeV}$ (and hence are not very fine-tuned) whereas the minimal GM model with multi-TeV squarks will have $\mu \gtrsim 1 \mathrm{TeV}$ (and thus is severely fine-tuned) so that Higgsinos can be detected (at a $\mathrm{TeV}$ linear collider) in the former models unlike in the latter 30 .

In the "more-minimal" supersymmetric models [14], the first and second generation squarks and sleptons (inlcuding right-handed sleptons, i.e., complete $\overline{\mathbf{5}}$ and $\mathbf{1 0}$ of $S U(5)$ ) are heavy, say about $10 \mathrm{TeV}$ so that the supersymmetric flavor problem is (partly) alleviated whereas the stops (and possibly the sbottom and stau) are lighter than $\sim 1 \mathrm{TeV}$ for naturalness (i.e., to keep $\mu \lesssim 500 \mathrm{GeV}$ ). So, the first and second generation squarks and sleptons are beyond the reach of LHC/TeV linear colliders while the top squarks should be detected at the LHC in these models 1. Thus, the collider signals of these "more-minimal" models are different from the models of sections 5.2 and 5.3 which have top (and other) squarks $\sim 1-3 \mathrm{TeV}$ (which might escape detection at the LHC) without resulting in fine-tuning, i.e., keeping $\mu \lesssim 500 \mathrm{GeV}$ and also (in some cases) all right-handed slepton masses $\sim 500 \mathrm{GeV}$.

\section{Summary}

In this paper, we have analysed whether in GMSB, the soft SUSY breaking mass spectrum can be such that the (up-type) Higgs (mass) ${ }^{2}$ (evaluated at the weak scale) is insensitive (or weakly sensitive) to the scalar (Higgs and stop) masses at the messenger scale (i.e., the boundary scalar masses) - this has been called "focussing" in [1] where supergravity mediation was considered. Then, the stop masses (and also other squark masses since they are related) can be multi$\mathrm{TeV}$ without leading to $m_{H_{u}}^{2} \sim-(\mathrm{TeV})^{2}$ at the weak scale (and the consequent fine-tuning in electroweak symmetry breaking).

In minimal GMSB, the Higgs mass at the boundary is too small compared to the stop masses

\footnotetext{
${ }^{30}$ Similarly, supergravity models with multi-TeV squarks, but without focussing, will have $\mu \gtrsim 1 \mathrm{TeV}$ and hence the Higgsinos will be beyond the reach of a $\mathrm{TeV}$ linear collider.

${ }^{31}$ Of course, the stops can also be multi-TeV in which case the model will be severely fine-tuned with $\mu \gtrsim 1$ $\mathrm{TeV}$ so that "Higgsinos" will be beyond the reach of TeV linear colliders.
} 


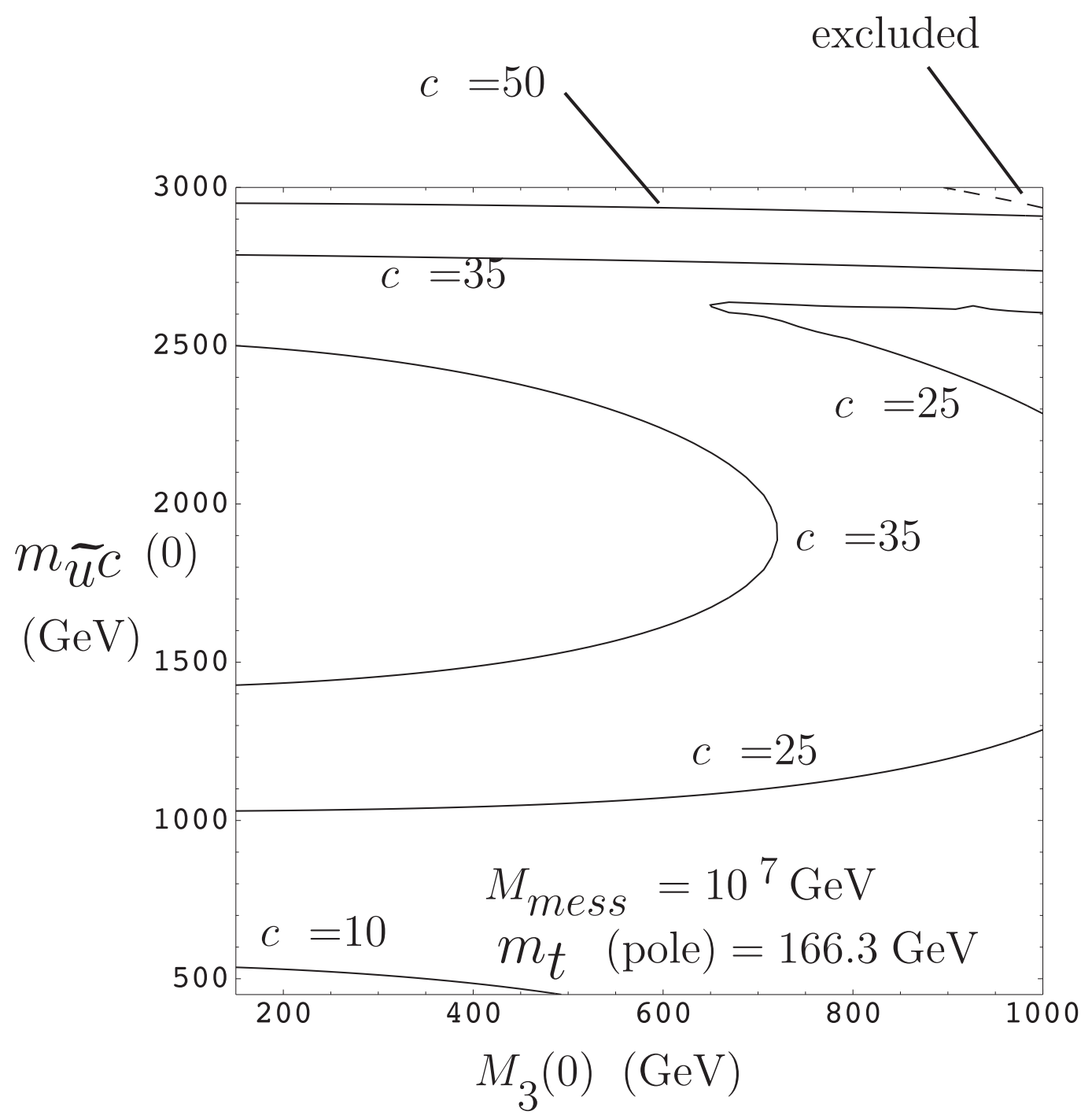

Figure 7: The fine-tuning $c$ (Eq. (20)) (solid lines) in the Dimopoulos, Thomas, Wells model (section 5.3) as a function of the right-handed up-squark mass $\left(m_{\tilde{u}^{c}}(0)\right)$ and the gluino mass $\left(M_{3}(0)\right)$ at the messenger scale. The messenger scale is $\approx 10^{7} \mathrm{GeV}, \tan \beta=10$ and the top quark (pole) mass is $166.3 \mathrm{GeV}$. The tiny region above the dashed line in the upper right corner has chargino mass smaller than $90 \mathrm{GeV}$ or $\mu^{2}<0$ and is therefore excluded. 


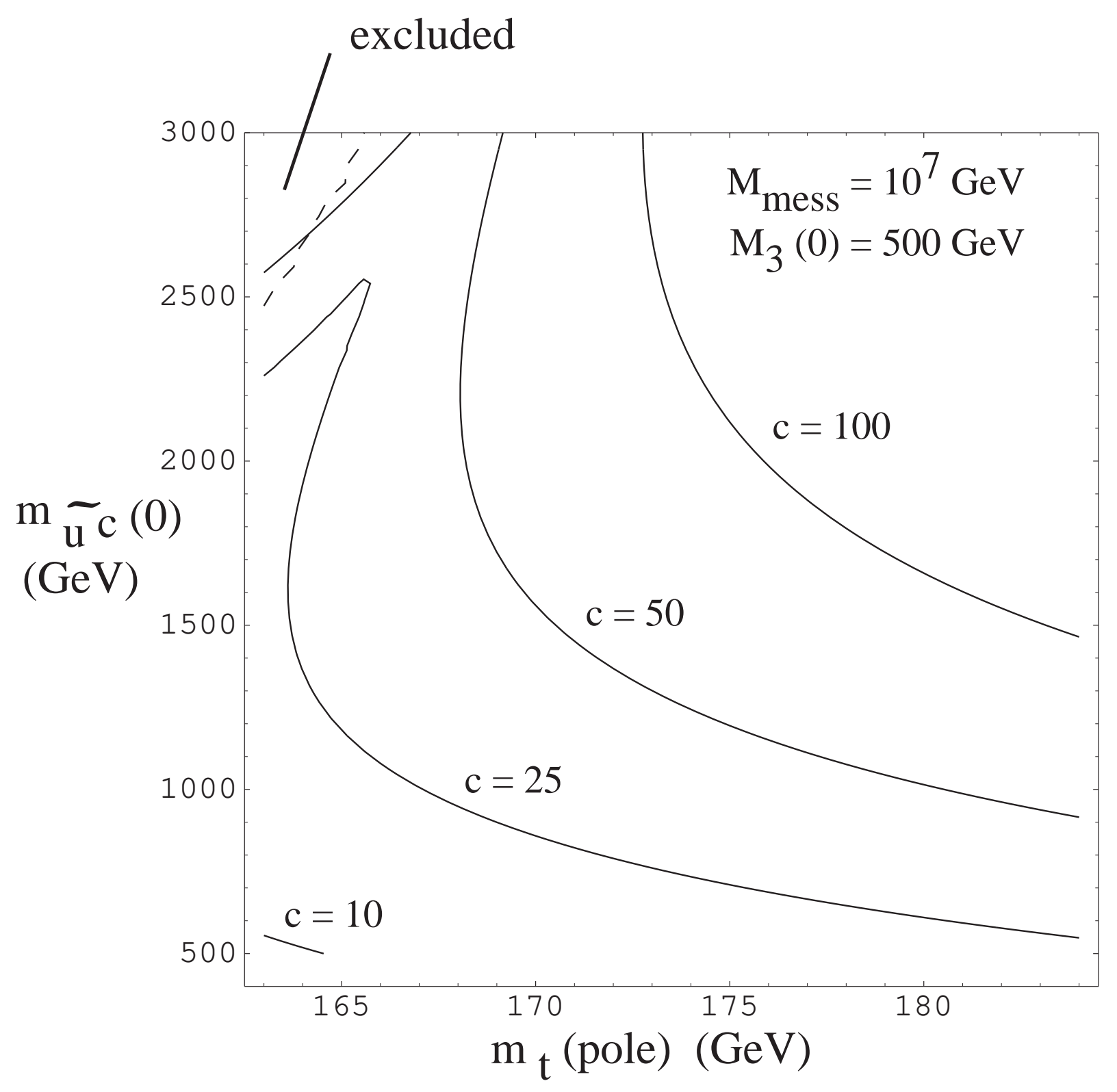

Figure 8: The fine-tuning $c$ (Eq. (20)) (solid lines) in the Dimopoulos, Thomas, Wells model (section 5.3) as a function of the right-handed up-squark mass at the messenger scale $\left(m_{\tilde{u}^{c}}(0)\right)$ and the top quark (pole) mass. The messenger scale is $\approx 10^{7} \mathrm{GeV}, \tan \beta=10$ and the gluino mass at the messenger scale, $M_{3}(0)$, is $500 \mathrm{GeV}$. The region to the left of the dashed line (the upper left corner) has chargino mass smaller than $90 \mathrm{GeV}$ or $\mu^{2}<0$ and is therefore excluded. 


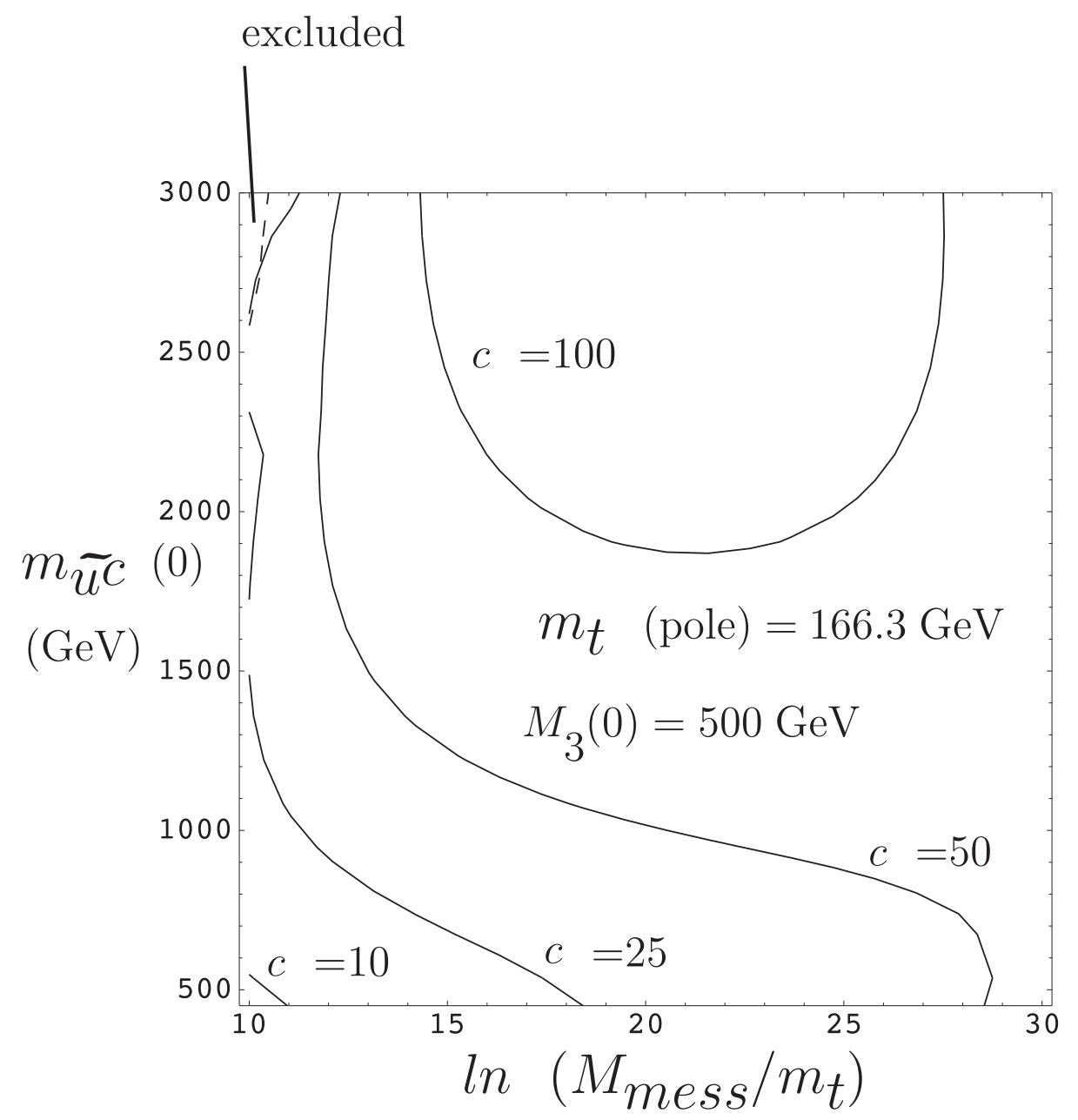

Figure 9: The fine-tuning $c$ (Eq. (20)) (solid lines) in the Dimopoulos, Thomas, Wells model (section 5.3) as a function of the right-handed up-squark mass at the messenger scale $\left(m_{\tilde{u}^{c}}(0)\right.$ ) and the messenger scale, $M_{\text {mess }}$. The gluino mass at the messenger scale, $M_{3}(0)$, is $500 \mathrm{GeV}$, $\tan \beta=10$ and the top quark (pole) mass is $166.3 \mathrm{GeV} \cdot \ln \left(M_{\text {mess }} / m_{t}\right)=10$ corresponds to $M_{\text {mess }} \approx 4 \times 10^{6} \mathrm{GeV}$ and $\ln \left(M_{\text {mess }} / m_{t}\right)=30$ corresponds to $M_{\text {mess }} \approx 2 \times 10^{15} \mathrm{GeV}$. The tiny region to the left of the dashed line (upper left corner) has chargino mass smaller than $90 \mathrm{GeV}$ or $\mu^{2}<0$ and is therefore excluded. 
so that this focussing does not happen. Also in minimal GM, the gaugino (especially the gluino) masses will also be multi-TeV if the scalars are multi-TeV, resulting in fine-tuning of $m_{Z}$.

Thus, in order for multi-TeV squarks to be natural in (non-minimal) models of GM, we require a) additional contribution to the Higgs mass (or a non-minimal mesenger sector) so that the ratio of the Higgs mass to the stop mass at the boundary can be higher (to achieve focussing) and b) suppression of gaugino masses (or a larger wino mass relative to gluino mass) so that even if the scalar masses are multi-TeV, the gaugino masses do not result in fine-tuning.

We discussed (general) ideas to satisfy these two requisites and also studied concrete models of GM which have multi-TeV $(\sim 1-3 \mathrm{TeV})$ squarks without leading to fine-tuning in EWSB. In some cases, these models have (in addition to $\sim 1-3 \mathrm{TeV}$ squarks) wino and gluino with a mass of $\sim 1 \mathrm{TeV}$ (without fine-tuning) and right-handed slepton mass $\sim 500 \mathrm{GeV}$ (so that righthanded sleptons can be detected at TeV lepton colliders) and (for very low messenger scales) lightest SM superpartner which decays inside the detector. Thus, the collider signals of these models can be different from the minimal supergravity models with multi-TeV squarks (and no fine-tuning) [ [] which have multi-TeV sleptons (which will escape detection at TeV lepton colliders) and $s u b-\mathrm{TeV}$ gauginos (and of course a stable lightest SM superpartner) so that it is possible to distinguish (experimentally) between the two scenarios. Unlike minimal GM models

with multi-TeV squarks (which have $\mu \gtrsim 1 \mathrm{TeV}$ ), the (non-minimal) models studied here can have $\mu \lesssim 500 \mathrm{GeV}$ so that the Higgsinos (to be precise the neutralino and chargino which have Higgsinos as the dominant component) can be detected at a $\mathrm{TeV}$ linear collider.

\section{References}

[1] J.L. Feng, K.T. Matchev, T. Moroi, hep-ph/9908309 and hep-ph/9909334.

[2] Review of Particle Physics, Particle Data Group, Eur. Phys. J. C3 (1998) 1.

[3] See, for example, G. Dvali, G.F. Giudice, A. Pomarol, hep-ph/9603238, Nucl. Phys. B478 (1996) 31 and the discussion of the effects of these additional Higgs soft masses in 13.

[4] B.A. Dobrescu, hep-ph/9703390, Phys. Lett. B403 (1997) 285.

[5] Z. Chacko et al., hep-ph/9704307, Phys. Rev. D56 (1997) 5466.

[6] K. Agashe, M. Graesser, hep-ph/9704206, Nucl. Phys. B507 (1997) 3.

[7] K. Agashe, M. Graesser, hep-ph/9801446, Phys. Rev. D59 (1999) 015007.

[8] See, for example, J. Ellis et al., Mod. Phys. Lett. A1 (1986) 57; R. Barbieri, G.F. Giudice, Nucl. Phys. B306 (1988) 63. 
[9] G.W. Anderson, D.J. Castaño, hep-ph/9409419, Phys. Lett. B347 (1995) 300.

[10] S. Raby, hep-ph/9702299, Phys. Rev. D56 (1997) 2852; hep-ph/9712254, Phys. Lett. B422 (1998) 158.

[11] S. Raby, K. Tobe, hep-ph/9807281, Nucl. Phys. B539 (1999) 3.

[12] S. Dimopoulos, F. Wilczek, Proceedings Erice Summer School, Ed. A. Zichichi (1981).

[13] S. Dimopoulos, S. Thomas, J.D. Wells, hep-ph/9609434, Nucl. Phys. B488 (1997) 39.

[14] A.G. Cohen, D.B. Kaplan, A.E. Nelson, hep-ph/9607394, Phys. Lett. B388 (1996) 588. For specific models which generate this hierarchy between the third and first two generations at the messenger scale, see, for example, G. Dvali, A. Pomarol, hep-ph/9607383, Phys. Rev. Lett. 77 (1996) 3728 and hep-ph/9708364, Nucl. Phys. B522 (1998) 3; R.N. Mohapatra, A. Riotto, hep-ph/9611273, Phys. Rev. D55 (1997) 4262; R-J. Zhang, hep-ph/9702333, Phys. Lett. B402 (1997) 101; A.E. Nelson, D. Wright, hep-ph/9702359, Phys. Rev. D56 (1997) 1598; H.P. Nilles, N. Polonsky, hep-ph/9707249, Phys. Lett. B412 (1997) 69; D.E. Kaplan et al., hep-ph/9806430, Phys. Rev. D60 (1999) 055003; J. Hisano, K. Kurosawa, Y. Nomura, hep-ph/9810411, Phys. Lett. B445 (1999) 316; D.E. Kaplan, G.D. Kribs, hep-ph/9906341 (D.E. Kaplan et al. and D.E. Kaplan, G.D. Kribs use "flavor" and gauge mediation to generate the soft SUSY breaking masses for the MSSM sparticles). This hierarchy may also be generated dynamically through RG evolution: see, for example, J. L. Feng, C. Kolda, N. Polonsky, hep-ph/9810500, Nucl. Phys. B546 (1999) 3. 